\title{
Nonequilibrium Multicritical Behaviour in Anisotropic Heisenberg Ferromagnet driven by Oscillating Magnetic Field
}

\author{
Muktish Acharyya \\ Department of Physics, Krishnanagar Government College, PO-Krishnanagar, Dist-Nadia, \\ PIN-741101, WB, India \\ E-mail: muktish@vsnl.net
}

\begin{abstract}
The Heisenberg ferromagnet (uniaxially anisotropic along z-direction), in the presence of time dependent (but uniform over space) magnetic field, is studied by Monte Carlo simulation. The time dependent magnetic field was taken as elliptically polarised in such a way that the resulting field vector rotates in the XZ-plane. In the limit of low anisotropy, the dynamical responses of the system are studied as functions of temperature and the amplitudes of the magnetic field. As the temperature decreases, it was found that the system undergoes multiple dynamical phase transitions. In this limit, the multiple transitions were studied in details and the phase diagram for this observed multicritical behavior was drawn in field amplitude and temperature plane. The natures (continuous/discontinuous) of the transitions are determined by the temperature variations of fourth-order Binder cumulant ratio and the distributions of order parameters near the transition points. The transitions are supported by finite size study. The temperature variations of the variances of dynamic order parameter components (for different system sizes) indicate the existence of diverging length scale near the dynamic transition points. The frequency dependences of the transition temperatures of the multiple dynamic transition are also studied briefly.
\end{abstract}

\section{INTRODUCTION}

The nonequilibrium dynamical phase transition [1], particularly in the kinetic Ising model, has drawn immense interest of the researchers working in the field of nonequilibrium statistical physics. The dynamic transition in the kinetic Ising model in the presence of sinusoidally oscillating magnetic field was noticed [2] first in the mean field solution of the dynamical equation for the average magnetisation. Due to the absence of fluctuations, in the mean field study, the dynamic transition would be possible also even in the static (zero frequency) limit. But the true dynamic transition should never occur in the static limit due to the presence of nontrivial thermodynamic fluctuations. The occurence of the true dynamic transitions for models, incorporating the thermodynamic fluctuations, was later shown in several Monte Carlo studies [1]. 
Since, the Ising model is quite simple and a prototype to study the nonequilibrium dynamical phase transitions, a considerable amount of work was done on it [1]. Among these, several studies were done to establish that all these transitions have some features which are similar to well known thermodynamic phase transitions. The divergence of "time scale" [3] and the "dynamic specific heat" [3] and the divergence of "length scale" [4] are two very important observations to establish that the dynamic transition, in kinetic Ising model driven by sinusoidally oscillating magnetic field, is indeed a thermodynamic phase transition.

Although, the dynamic phase transition in kinetic Ising model is an interesting phenomenon which acts as a simple example to grasp the various features of nonequilibrium phase transitions, it has several limitations. Since the orientations of the spins are limited by only two (up/down) directions, some interesting features of dynamic transitions (related to the dynamical transverse ordering) cannot be observed in Ising model. The classical vector spin model [5] would be the proper choice to study such interesting phenomena which are missing in Ising model. The "off-axial" dynamic transition [6], recently observed in Heisenberg ferromagnet, is an example of such interesting phenomena. In the "off-axial" dynamic transition, the dynamical symmetry along the axis of anisotropy can be broken dynamically by applying an oscillating magnetic field along any perpendicular direction. There are several important studies done on classical vector spin models driven by oscillating magnetic field. The dynamic phase transition in an anisotropic XY ferromagnet driven by oscillating magnetic field was recently studied [7] by solving Ginzburg-Landau equation. The dynamic phase transition and the dependence of its behaviour on the bilinear exchange anisotropy of classical Heisenberg ferromagnet (thin film), was studied by [8] Monte Carlo Simulation. The dynamic transitions alongwith hysteresis scaling in Heisenberg ferromagnet was also studied [9] both by Monte Carlo simulation and mean field solution.

All these studies (discussed in above paragraph) of dynamic transitions in classical vector spin model give single dynamic transitions. There are a few studies in Heisenberg ferromagnets where the multiple dynamic transition was reported [10,11]. A recent study [10] of dynamical phase transition in thin Heisenberg ferromagnetic films with bilinear exchange anisotropy has shown multiple phase transitions for the surface and bulk layers of the film at different temperatures. However, another study [11] shows triple dynamic transitions (at three different temperatures), for bulk dynamic ordering only, in uniaxially anisotropic Heisenberg ferromagnet driven by elliptically polarised magnetic field. In the present paper, the multiple dynamic transition (bulk only) [11] in uniaxially anisotropic Heisenberg ferromagnets driven by elliptically polarised magnetic field, is studied extensively.

In this paper, the multiple dynamic transition, in the uniaxially anisotropic classical Heisenberg ferromagnet in presence of elliptically polarised magnetic field, is studied by Monte Carlo simulation. The various dynamical phases were found. The multiple dynamic phase transition was observed by studying the temperature variation of the dynamic specific heat and the derivatives of dynamic order parameter components. The nature (continuous/discontinuous) of the transition was detected by studying the distribution of order parameter and the temperature variation of Binder cumulant ratio near the transition point. A finite size study was done supporting the multiple dynamic phase transitions. A length scale was found to diverge near the dynamic transition points. And finally and most importantly, the phase diagram for this multiple dynamic phase transition was drawn. The frequency dependence of these multiple dynamic transitions was also studied briefly. 
The paper is organized as follows: in the next section the description of the system, i.e., the Heisenberg ferromagnet in polarised magnetic field, is given, section III describes the Monte Carlo simulation technique employed here to study the dynamical steady state of this type of classical vector spin model, in detail. The numerical results with diagrams are reported in section IV and the paper ends with a summary in section V.

\section{HEISENBERG FERROMAGNET IN POLARIZED MAGNETIC FIELD}

The classical anisotropic (uniaxial and single-site) Heisenberg model, with nearest neighbour ferromagnetic interaction in the presence of a magnetic field can be described by the following Hamiltonian:

$$
H=-J \Sigma_{<i j>} \mathbf{S}_{\mathbf{i}} \cdot \mathbf{S}_{\mathbf{j}}-D \Sigma_{i}\left(S_{i z}\right)^{2}-\mathbf{h} \cdot \Sigma_{i} \mathbf{S}_{\mathbf{i}}
$$

In the above expression, $\mathbf{S}_{\mathbf{i}}\left[S_{i x}, S_{i y}, S_{i z}\right]$ represents a classical spin vector (situated at the $i$ th lattice site) of magnitude unity, i.e., $|\mathbf{S}|=1$ or $S_{i x}^{2}+S_{i y}^{2}+S_{i z}^{2}=1$. The classical spin vector $\mathbf{S}_{\mathbf{i}}$ may take any (unrestricted) angular orientation in the vector spin space. The first term, in the Hamiltonian, represents the nearest -neighbour $(<i j\rangle)$ ferromagnetic $(J>0)$ interaction. The factor $D>0$, in the second term, represents the strength of uniaxial ( $z$ axis here) anisotropy which is favoring the spin vector to be aligned along the $z$ axis. This is readily seen since the second term minimizes energy for maximization of the value of $S_{i}^{z}$. Here, it may be noted that for $D \rightarrow \infty$ the system goes to the Ising limit and for $D=0$ the system is in the isotropic Heisenberg limit. The last term stands for the interaction with the externally applied time dependent magnetic field $\left[\mathbf{h}\left(h_{x}, h_{y}, h_{z}\right)\right]$. The magnetic-field components are sinusoidally oscillating in time, i.e., $h_{\alpha}=h_{0 \alpha} \cos (\omega t)$, where $h_{0 \alpha}$ is the amplitude and $\omega$ is the angular frequency ( $\omega=2 \pi f ; f$ is the frequency) of the $\alpha$ th component of the magnetic field. In the present study, the field is taken elliptically polarised. In general, the field vector is represented as

$$
\mathbf{h}=\hat{x} h_{x}+\hat{y} h_{y}+\hat{z} h_{z}=\hat{x} h_{0 x} \cos (\omega t)+\hat{z} h_{0 z} \sin (\omega t) .
$$

The time eliminated relation between $h_{x}\left(=h_{0 x} \cos (\omega t)\right)$ and $h_{z}\left(=h_{0 z} \sin (\omega t)\right.$ is

$$
\frac{h_{x}^{2}}{h_{0 x}^{2}}+\frac{h_{z}^{2}}{h_{0 z}^{2}}=1 .
$$

This is the equation of an ellipse which indicates that the magnetic field vector is elliptically polarised (for $h_{0 x} \neq h_{0 z}$ ) and lies on the $X-Z$ plane. For $h_{0 x}=h_{0 z}=h_{0}$ (say), the field will be circularly polarised and $h_{x}^{2}+h_{z}^{2}=h_{0}^{2}$. The magnetic fields and the strength of anisotropy $D$ are measured in units of $J$. The model is defined on a simple cubic lattice of linear size $L$ with periodic boundary conditions applied in all three $(x, y, z)$ directions.

\section{MONTE CARLO SIMULATION METHOD}

Monte Carlo (MC) simulation method was employed to study the above described model. The algorithm is [12] described below. The system is slowly cooled down from a random initial spin configuration [13], to obtain the steady state spin configuration at a particular temperature 
$T$ (measured in units of $J / k_{B}$, where $k_{B}$ is the Boltzmann constant). The initial random spin configuration was generated as follows $[6,13]$ : two different (uncorrelated) random numbers $r_{1}$ and $r_{2}$ (uniformly distributed between -1 and +1 ), are chosen in such a way that $R^{2}=r_{1}^{2}+r_{2}^{2}$ becomes less than or equal to unity. The set of values of $r_{1}$ and $r_{2}$, for which $R^{2}>1$, are rejected. Now, $S_{i x}=2 u r_{1}, S_{i y}=2 u r_{2}$ and $S_{i z}=1-2 R^{2}$, where $u=\sqrt{1-R^{2}}$. After preparing initial random configuration of spins (this is the proper choice of the spin configurations, corresponding to very high temperature), one has to find a steady state configuration for any fixed temperature $T$. For any fixed set of values of $h_{0 x}, h_{0 z}, \omega$ and $D$ and at any particular temperature $T$, a lattice site $i$ has been chosen randomly (random updateing scheme). The value (random direction) of the spin vector at this randomly chosen site is $\mathbf{S}_{\mathbf{i}}$ (say). The energy of the system is given by the Hamiltonian (Eq. 1). A test spin vector $\mathbf{S}_{\mathbf{i}}^{\prime}$ is then chosen (for a trial move) at any random direction (following the same algorithm described above). For this choice of $\mathbf{S}_{\mathbf{i}}^{\prime}$, at site $i$ the energy will be $H=-J \Sigma_{<i j>} \mathbf{S}_{\mathbf{i}}^{\prime} \cdot \mathbf{S}_{\mathbf{j}}-D \Sigma_{i}\left(S_{i z}^{\prime}\right)^{2}-\mathbf{h} \cdot \Sigma_{i} \mathbf{S}_{\mathbf{i}}^{\prime}$. The change in energy, associated with this change in the direction of spin vector (from $\mathbf{S}_{\mathbf{i}}$ to $\mathbf{S}_{\mathbf{i}}^{\prime}$ at lattice site $i$ ), is $\Delta H=H^{\prime}-H$. The Monte Carlo method [12] will decide whether this trial move is acceptable or not. The probability of this move (chosen here) is given by Metropolis rate [14]

$$
W\left(\mathbf{S}_{\mathbf{i}} \rightarrow \mathbf{S}_{\mathbf{i}}^{\prime}\right)=\operatorname{Min}\left[1, \exp \left(-\frac{\Delta H}{k_{B} T}\right)\right] .
$$

Now, this probability will be compared with a random number $R_{p}$ (say) (uniformly distributed between zero and one). If $R_{p}$ does not exceed $W$, the move $\left(\mathbf{S}_{\mathbf{i}} \rightarrow \mathbf{S}_{\mathbf{i}}^{\prime}\right)$ will be accepted. In this way the spin vector $\mathbf{S}_{\mathbf{i}}$ is updated. $L^{3}$ numbers of such random updates of spins, defines one Monte Carlo step per site (MCSS) and this is considered as the unit of time in this simulation. The efficiency of the MC technique can be increased by choosing the direction of the spin for the trial move close to the present direction which will increase the probability of acceptance. So, 50 MCSS are required to have one complete cycle of the oscillating magnetic field (of frequency $f=0.02)$ and hence the time period $(\tau)$ of the field becomes 50 MCSS. Any time dependent macroscopic quantity (i.e., any component of magnetisation at any instant $t$ ) is calculated as follows: Starting with an initial random spin configuration (corresponding to the high-temperature disordered phase), the system is allowed to become stable (dynamically) up to $5 \times 10^{4}$ MCSS (i.e., 1000 complete cycle of the oscillating field). The average value of various physical quantities are calculated from further $5 \times 10^{4}$ MCSS (i.e., averaged over another 1000 cycles). This was checked carefully that the number of MCSS mentioned above is sufficient to achieve dynamical steady state value of the measurable quantities, etc. which would clearly show the dynamic transition points within limited accuracy. But to describe the critical behaviour very precisely (i.e., to estimate critical exponents etc) a much longer run is required. The total length of the simulation becomes $10^{5}$ MCSS. The system is slowly cooled down ( $T$ has been reduced by a small interval $\delta T=0.02$ here) to get the values of the statistical quantities in the lower temperature phase. Here, the last spin configuration corresponding to the previous temperature is employed to act as initial configuration for the new (lower) temperature. The CPU time required for $10^{5}$ MCSS is approximately $30 \mathrm{~min}$ on an Intel Pentium-III processor.

One important point may be noted here regarding the dynamics chosen in this simulational study. Since the spin components do not commute with the Heisenberg Hamiltonian, it has intrinsic quantum mechanical dynamics. Considering the intrinsic dynamics, there was a study [15] of the structure factor and transport properties in XY model. However, the present pa- 
per aims to study the nonequilibrium phase transition governed by thermal fluctuations only. Keeping this in mind, one should choose the dynamics that arising solely only due to the interaction with thermal bath. Since the objective is different, in this paper, the dynamics chosen (arise solely due to the interaction with a thermal bath) were Metropolis dynamics. The effect of intrinsic spin dynamics is therefore not considered here.

\section{NUMERICAL RESULTS}

The Monte Carlo simulations, in the present study, were done on a simple cubic lattice of linear size $L=20$. For a fixed set of values of amplitudes $\left(h_{0 x}, h_{0 z}\right)$ and frequency $(f)$ of polarised magnetic field, strength of anisotropy $(D)$ and temperature $(T)$, the instantaneous magnetisation components (per lattice site) were calculated as follows: $m_{x}(t)=\Sigma_{i} S_{i}^{x} / L^{3}$, $m_{y}(t)=\Sigma_{i} S_{i}^{y} / L^{3}$ and $m_{z}(t)=\Sigma_{i} S_{i}^{z} / L^{3}$. The dynamic order parameter is defined as the time averaged magnetisation over a full cycle of the oscillating magnetic field. In this case the dynamic order parameter $\mathbf{Q}$ is a vector (since the magnetisation is vector). The components of the dynamic order parameter are calculated as $Q_{x}=(1 / \tau) \oint m_{x}(t) d t, Q_{y}=(1 / \tau) \oint m_{y}(t) d t$ and $Q_{z}=(1 / \tau) \oint m_{z}(t) d t$. Here, four different dynamical phases are identified. The high temperature disordered phase is denoted as $P_{0}:\left(Q_{x}=Q_{y}=Q_{z}=0\right)$. Other three ordered phases are $P_{1}:\left(Q_{x}=0, Q_{y} \neq 0, Q_{z}=0\right), P_{2}:\left(Q_{x} \neq 0, Q_{y}=0, Q_{z} \neq 0\right)$ and $P_{3}:\left(Q_{x}=\right.$ $\left.0, Q_{y}=0, Q_{z} \neq 0\right)$. The phase diagram, obtained from multiple dynamic transitions, is plotted in $h_{0 x}-T$ plane and is shown in fig 1 . The methods of getting the phase boundaries are discussed in the following paragraphs.

The signature of such successive phase transitions are also observed by studying the temperature variations of the magnitude of the dynamic order parameter, energy, specific heat and the temperature derivatives of dynamic order parameter components. For the same set of values $D=0.2, h_{0 x}=0.3$ and $h_{0 z}=1.0$ the temperature variations of various dynamic quantities are studied and shown in Fig. 2. The temperature variations of the dynamic order parameter components $Q_{x}, Q_{y}$ and $Q_{z}$ are shown in Fig. 2(a). As the system is cooled down, from a high-temperature dynamically disordered $(\mathbf{Q}=\overrightarrow{0})$ phase, it was observed that first the system undergoes a transition from dynamically disordered $P_{0}$ phase $(\mathbf{Q}=\overrightarrow{0})$ to a dynamically Y-ordered ( $Q_{y} \neq 0$ only) phase $P_{1}$ and the transition temperature is $T_{c 1}$ (say). It may be noted here that the resultant vector of elliptically polarised magnetic field lies in the $x-z$ plane and the dynamic ordering occurs along the $y$-direction only $\left(Q_{x}=0, Q_{y} \neq 0, Q_{z}=0\right)$. This is clearly an off-axial transition [6]. In the case of this type of off-axial transition the dynamical symmetry (in any direction; $y$ here) is broken by the application of the magnetic field in the perpendicular (lies in the $x-z$ plane here) direction. As the system cools down further, this phase $P_{1}$ persists over a considerable range of temperature and at a temperature $T_{c 2}$ a second transition was observed. In this phase, the system becomes dynamically ordered both in the $X$ and $Z$ directions at the cost of $Y$ ordering. Usually one gets the dynamically ordered phase of second kind $P_{2}:\left(Q_{x} \neq 0, Q_{y}=0, Q_{z} \neq 0\right)$. Here, the dynamical ordering is planar (lies on $x-z$ plane) and the dynamical ordering occurs in the same plane on which the field vector lies. This transition is not of off-axial type. As the temperature decreases further one ends up with a low-temperature dynamically ordered phase of third kind $P_{3}:\left(Q_{x}=0, Q_{y}=0, Q_{z} \neq 0\right)$ via a transition occurs at temperature $T_{c 3}$. The system continues to increase the dynamical 
$Z$ ordering $\left(Q_{z} \neq 0\right.$ only) as the temperature decreases further. The low-temperature phase is only dynamically $Z$ ordered. No further transition was observed as one cools the system further. From Fig.2(a), it is quite clear, if one observes carefully, that the sizes of the errorbars of $Q_{x}, Q_{y}$ and $Q_{z}$ are maximal near the transition points, indicating the growth of fluctuations near the transiton points.

One gets qualitative ideas of multiple dynamic phase transitions from the above mentioned studies. However, to estimate precisely the transition temperatures $T_{c 1}, T_{c 2}$ and $T_{c 3}$ further studies are required. For this reason, the temperature variations of the derivatives (with respect to temperature) of the dynamic order parameter components are studied. The derivatives were calculated numerically by using central difference formula [16]

$$
\frac{d f(x)}{d x}=\frac{f(x+\delta x)-f(x-\delta x)}{2 \delta x} .
$$

Fig. 2(b) shows such variations studied as a function of temperature. The derivative $d Q_{y} / d T$, shows a sharp minimum at $T=T_{c 1} \simeq 1.22$. The second transition temperature $T_{c 2}$ was estimated from the position of sharp maximum of $d Q_{y} / d T$ and the corresponding sharp minima of $d Q_{x} / d T$ and $d Q_{z} / d T$. This gives $T_{c 2} \simeq 0.96$. At slightly lower temperature than $T_{c 2}$, one observes another maximum of $d Q_{x} / d T$ and minimum of $d Q_{z} / d T$ both at the same position $T=T_{c 3} \simeq 0.88$. From this study one gets the quantitative measure of the transition temperatures of multiple dynamic transition [11]. The maximum error, in estimating the transition temperatures, is 0.01 .

Similar values of the transition temperatures $\left(T_{c 1}, T_{c 2}, T_{c 3}\right)$ for the multiple dynamic transition can be estimated independently from the study of the temperature variations of dynamic energy and specific heat. The dynamical energy $(E)$ is defined as the time averaged value of the instantaneous energy over a full cycle of the oscillating magnetic field. From the definition, $E=(1 / \tau) \oint H d t$ ( $H$ is given in Eqn. 1). The temperature variation of $E$ is studied and shown in Fig. 2(c). This shows two inflection points and one (the middle one) discontinuity at the same location of transition temperatures estimated and mentioned above. This will be very clear if one studies the derivative of the dynamic energy, namely the dynamic specific heat $C=d E / d T$ (calculated by using central difference formula 5). The temperature variation of dynamic specific heat $C$ was studied and shown in Fig. 2(d). It indicates three peaks at three different temperatures supporting $T_{c 1} \simeq 1.22, T_{c 2} \simeq 0.96$ and $T_{c 3} \simeq 0.88$. Thus the estimation of transition temperatures for the multiple dynamic transition was reexamined by another independent study. The study of the temperature variation of dynamic specific heat has another importance. It independently supports multiple transitions as well as it indicates that these transitions are indeed thermodynamic phase transitions. Now one may employ both the methods of studying the temperature variations of derivatives of dynamic order parameter components and the dynamic specific heat to estimate the transition points. One gets three different dynamic transitions for the parameter values $D=0.2, h_{0 x}=0.3$ and $h_{0 z}=1.0$. This three transitions senario is observed for a range of values of $h_{0 x}$ (keeping other parameters fixed $D=0.2$ and $h_{0 z}=1.0$ ) between $h_{0 x}=0.1$ to $h_{0 x}=0.5$ (within the precision considered here). The temperature variations of dynamic specific heat $C$ are shown in fig 3 for two different values of $h_{0 x}(=0.1$ and 0.5$)$. Both show the three dynamic transitions. It may be noted from fig. 3 that the transition temperature decreases as the amplitude $h_{0 x}$ increases. Now, let us see what happens if one takes the value of the $x$-amplitude of elliptically polarised magnetic field, 
i.e., $h_{0 x}$, outside this range, keeping all other parameter values unchanged.

For $h_{0 x}=0.0$ (effectively the field is now linearly polarised along $z$ direction only), to estimate the transition points the temperature variations of dynamic specific heat was studied and plotted in fig. 4(a). This shows two distinct and well separated peaks indicating two dynamic transitions, one at $T \simeq 1.24$ and other at $T \simeq 0.98$. Now to characterise the different phases the temperature variations of dynamic order parameter components were studied. This study is shown in fig. 4(b). This shows that the system starts to get dynamically ordered from a high-temperature disordered phase. So, the high-temperature ordered phase $\left(Q_{x} \neq 0\right.$, $\left.Q_{y} \neq 0, Q_{z}=0\right)$ is quite different from $P_{1}$ (described above for $h_{0 x} \neq 0$ ). The second (lowtemperature ordered phase) is $P_{3}$ type. This transition (from high-temperature ordered phase to low-temperature ordered phase) occurs at $T_{c 3} \simeq 0.98$. So, one gets two distinct transitions for $D=0.2, h_{0 x}=0.0$ and $h_{0 z}=1.0$. It may be noted here that the temperature variations of the order parameter components, mainly $Q_{x}$ and $Q_{y}$, are quite scattered (near the low-temperature transition). Here, $Q_{x}$ and $Q_{y}$ are calculated by averaging over 2000 cycles discarding first 2000 cycles $\left(10^{5} M C S S\right)$. For this reason the derivatives $d Q_{x} / d T$ and $d Q_{y} / d T$ do not give smooth variation with respect to temperature and are not shown.

Now if the value of $h_{0 x}$ is higher (say around $h_{0 x}=0.6$ ) with all other parameter values fixed (i.e., $f=0.02, D=0.2$ and $h_{0 z}=1.0$ ) the triple dynamic transitions (observed for $h_{0 x}=0.3$ ) reduces to double transitions. It gives two distinct peaks of $C$ indicating two different dynamic transitions at $T \simeq 1.20$ and $T \simeq 0.82$. So, in this case one gets two dynamically ordered phases, namely $P_{1}$ (higher temperature ordered phase) and $P_{3}$ (lower temperature ordered phase). The transition from $P_{1}$ phase to $P_{3}$ phase occurs at $T \simeq 0.82$. The high-temperature ordered phase $\left(P_{1}\right)$ transition (from dynamically disordered phase) occurs at $T \simeq 1.20$. These two transitions were reexamined by studying the temperature variations of the derivatives of the dynamic order parameter and obtained the same transition temperatures.

From the above discussion it is quite clear that if one studies the dynamic phase transitions by varying $h_{0 x}$ only (keeping $D=0.2$ and $h_{0 z}=1.0$ fixed), one would get two transitions for $h_{0 x}=0.0$. Just above $h_{0 x}=0.0$, i.e., starting from $h_{0 x}=0.1$ upto $h_{0 x}=0.5$ one would get three transitions (and three ordered phases). Above this value, say $h_{0 x}=0.6$ one would get again two transitions (two ordered phases). If one continues further, it was observed that the two transitions feature continues. The transition points (temperatures) are estimated by studying specific heat and the derivatives of the dynamic order parameter components. These are not shown in figure, only the results (obtained from the peak positions of the specific heat and positions of maximum or minimum of $\left.d Q_{\alpha} / d T\right)$ are taken. It was observed further that all the transition points shift towards lower temperatures for higher values of $h_{0 x}$. The whole results of these multiple transitions temperatures and plotted in $T-h_{0 x}$ plane (fig. 1) to get the multiple dynamic phase boundary.

In the phase diagram (fig. 1), the outermost boundary separtes the dynamically $Y$-ordered phase $P_{1}:\left(Q_{x}=0, Q_{y} \neq 0, Q_{z}=0\right)$ from the disordered phase $P_{0}:\left(Q_{x}=0, Q_{y}=0, Q_{z}=0\right)$. The transition temperature decreases as the value of the field amplitude $h_{0 x}$ increases. For lower values of $h_{0 x}$, the region bounded by the boundaries marked by the symbols circle and bullet is the ordered phase characterised as $P_{2}:\left(Q_{x} \neq 0, Q_{y}=0, Q_{z} \neq 0\right)$. This region is quite small (in area) but very clear and was observed very carefully. The transition temperatures for both the boundaries (left and right) of this phase decrease as the field amplitude $h_{0 x}$ increases. The innermost (in the $T-h_{0 x}$ plane) region, whose boundary is partly marked by circle (for 
higher values of $h_{0 x}$ ) and partly marked by bullet (for lower values of $h_{0 x}$ ), represents the lowtemperature phase characterised by $P_{3}:\left(Q_{x}=0, Q_{y}=0, Q_{z} \neq 0\right)$. Here also, the transition temperature decreases as the value of the field amplitude $h_{0 x}$ increases.

The question which should arise now, what will be the nature (continuous/discontinuous) of these multiple dynamic transitions ? To get the answer of this questions, the distributions of the magnitudes of the dynamic order parameter components are studied near the transition points. On the phase boundary (Fig.1), a particular value of $h_{0 x}(=0.7)$ is chosen. This choice is not arbitrary. In this region, the phase boundaries are well separated to study comfortably the temperature variations of fourth-order Binder cumulant ratio [17] $U_{y}=1-<Q_{y}^{4}>/(3<$ $Q_{y}^{2}>^{2}$ ). Since the transition from $P_{0}$ to $P_{1}$ phase is indicated by $Q_{y}$ the distribution of the magnitude of $Q_{y}$ is studied near the transition point. The histogram is shown in Fig.5 for four different temperatures around the transition temperature. This figure shows that as one goes through the results of temperature from $T=1.16$ to that of higher temperature, the peak of the distributions shifts towards $Q_{y}=0$ continuously and around $T=1.20$ this gets peaked near $Q_{y} \simeq 0$. For slightly higher value of the temperature, say $T=1.22$, the distribution is peaked around $Q_{y}=0$, corresponding to the disordered phase. This indicates the transition is continuous with $T_{c 1} \simeq 1.20$ (for the transition from $P_{0}$ to $P_{1}$ phase) and one could guess it from the temperature variation of $Q_{y}$ and $d Q_{y} / d T$. This same technique was applied to study the nature of the transition from $P_{1}$ to $P_{3}$ phase. The distribution of $Q_{y}$ is shown (in Fig.6) for three different temperatures. Here, the different (from the previous case just discussed above) senario was observed and may be noted, that the value of $Q_{y}$ drops to zero from a nonzero value as the system gets cooled. At $T=0.80$ the distribution is singly peaked near $Q_{y} \simeq 0.63$. At slightly lower temperature $T=0.79$, the distribution get doubly peaked. One additional peak appears near $Q_{y} \simeq 0$. This simultaneous appearance of two peaks at very close to the transition temperature is a clear signature of discontinuous transition with $T_{c} \simeq 0.79$ (for the transition from $P_{1}$ to $P_{3}$ phase). From this study, one can get the idea about the maximum error $(0.01$ here) in estimation of the transition temperature. As one lowers the temperatures, $T=0.78$, one gets only one peak of the distribution around $Q_{y}=0$ after the transition. Here also, this could be guessed from the temperature variation of $Q_{y}$. These studies indicate that the hightemperature transition is a continuous one and the low-temperature transition is discontinuous type. These natures of the transitions were reexamined by studying the temperature variation of Binder cumulant ratio $U_{y}$. For a continuous transition, $U_{y}$ should change monotonically from 0 to $2 / 3$ as one tunes the system from the disordered to the ordered phase. On the other hand, for a discontinuous transition, $U_{y}$ develops sharp minimum, whose location corresponds to the transition point. The results, obtained here, indeed show this (Fig.7). Fig.7 shows, that as one tunes the system from high temperature, the Binder cumulant ratio $U_{y}$ first grows monotonically from 0 to $2 / 3$ around $T_{c 1} \simeq 1.20$ indicating that the high-temperature transition is continuous. As the temperature decreases further, it shows a very sharp minimum around $T_{c} \simeq 0.80$ (for the transition from $P_{1}$ to $P_{3}$ phase) indicating the discontinuous nature of the low-temperature transition. These two (multi-histogram analysis and Binder cumulant ratio) studies together confirm that the high-temperature transition is a continuous type and the low-temperature transition is discontinuous type. Since, the evidence of the nature of the hightemperature transition is found to be of continuous type, it will be quite interesting to study the scaling behaviour (if any) with the estimations of critical exponents. But for this, one has to estimate the transition temperatures more precisely which will require larger sizes of the 
system.

What will be the nature of the transition from $P_{2}$ to $P_{3}$ phase ? For lower values of $h_{0 x}$ $(=0.3$ chosen here) one would be able to see this transition. From Fig.2(a), this transition is indicated by the temperature variation of $Q_{z}$. Since, this transition corresponds to very small change of $Q_{z}$, it is quite difficult to observe any change of the distribution of $Q_{z}$ near the transition temperature. However, the temperature variation of the Binder cumulant ratio $U_{z}=1-<Q_{z}^{4}>/\left(3<Q_{z}^{2}>^{2}\right)$ indeed shows (see Fig.8) smooth variation near the transition point and it grows monotonically from 0 to $2 / 3$ which indicates the transition is continuous with $T_{c 3} \simeq 0.88$ (for the transition from $P_{2}$ to $P_{3}$ phase). All these natures of the transitions are marked in the phase diagram (Fig.1).

The finite size study is also done to confirm that the observation is not an artifact of limited size of the system. This study was done for a particular set $\left(f=0.02, D=0.2, h_{0 x}=0.3, h_{0 z}=\right.$ 1.0) of values of the parameters. Different statistical quantities were studied as functions of temperatures for different linear sizes ( $L=10,20,30$ here) of the system. This particular choice of the values of $D, h_{0 x}$ and $h_{0 z}$ is meaningful in the sense that the three transitions phenomenon was observed clearly for this particular values of parameters and the most important part of the phase boundary. Figure 9 shows the temperature variations of $d Q_{\alpha} / d T$ for $L=10,20$ and 30. From the figure it is clear that both the sharpness and the height (depth) of maximum (minimum) indicating the transition points increase as the system size increase. The dynamic specific heat is also studied as a function of temperature for different $L$ and same set of other parameter values. This is shown in fig. 10. This also indicates that the height of the peaks (indicating the transitions) increases as the system size increases. The finite size study, done and briefly reported here, at least may indicate that the multiple dynamic transitions mentioned above is not an artifact of finite size effect.

The variances of the dynamic order parameter components i.e., $L^{3} \operatorname{Var}\left(Q_{\alpha}\right)=L^{3}\left[<Q_{\alpha}^{2}>\right.$ $\left.-<Q_{\alpha}>^{2}\right]=L^{3} \delta Q_{\alpha}^{2}$ are studied as function of temperature and for different system sizes. The values of other parameters are $f=0.02, D=0.2, h_{0 x}=0.3$ and $h_{0 z}=1.0$. Figure $11(\mathrm{a})$ shows temperature variation of $L^{3} \operatorname{Var}\left(Q_{x}\right)=L^{3}\left[<Q_{x}^{2}>-<Q_{x}>^{2}\right]=L^{3} \delta Q_{x}^{2}$ for $L=10,20$ and 30. It gets sharply peaked at around $T=T_{c 2} \simeq 0.96$ (the transition temperature from $P_{1}$ phase to $P_{2}$ phase). The height of the peak increases for larger system sizes. In fig. 11(b) the $L^{3} \operatorname{Var}\left(Q_{y}\right)$ is plotted against temperature for different system sizes. It shows two peaks. The high-temperature peak (very short in height but visible in this scale) is located at $T \simeq 1.22$. This corresponds to the transition from disordered $\left(P_{0}\right)$ to first Y-ordered $\left(P_{1}\right)$ phase. The lowtemperature peaks are positioned at around $T \simeq 0.96$ corresponding to the transition from $P_{1}$ to $P_{2}$. Here, also the heights of the peaks increases systematically as the system size increases. Similar things are observed for $L^{3} \operatorname{Var}\left(Q_{z}\right)$ and shown in fig. 11(c). Here, an important thing should be noted that this study indicates that their exists a diverging length scale at the dynamic transition points [4], for the multiple dynamic transitions in this system. It should also be noted that the transition (from $P_{2}$ to $P_{3}$ ) temperature $T_{c 3}$ cannot be resoved from $T_{c 2}$ in the present study. It was already indicated by the results shown in Fig.2(a). The sizes of the errorbars of $Q_{x}, Q_{y}$ and $Q_{z}$ becomes maximum near $T_{c 1}$ and $T_{c 2}$. However, the growth of the errorbars of $Q_{z}$ is not quite clear near $T_{c 3}$.

The frequency dependence of these multiple dynamic transitions are also studied very briefly. For a lower frequency $(f=0.01)$ of the polarised magnetic field, the multiple dynamic transition was studied with $D=0.2, h_{0 x}=0.3 h_{0 z}=1.0$. The temperature variations of the magnitudes 
of the dynamic order parameter components are plotted in Fig.12(a). This shows similar kind of temperature variations as that was obtained for $f=0.02\left(D=0.2, h_{0 x}=0.3\right.$ and $h_{0 z}=1.0$ ), with considerable shifting of transition points towards lower temperatures. From the study of the temperature variations of the derivatives of the dynamic order parameter components (shown in Fig.12(b)) one gets $T_{c 1} \simeq 1.06, T_{c 2} \simeq 0.76$ and $T_{c 3} \simeq 0.62$. A similar study was done for higher values of $h_{0 x}(=1.5)\left(f=0.01, D=0.2\right.$ and $\left.h_{0 z}=1.0\right)$. The results are shown in Fig.13. In this case only two transitions were observed and the transition temperatures estimated are $T_{c 1} \simeq 0.60$ and $T_{c 3} \simeq 0.30$. From these studies, one can get the indication that as the frequency decreases (or as one approaches the static limit) the transition points shifts towards lower temperatures keeping the qualitative nature of the phase diagram (for $f=0.02$ ) unchanged. This is quite expected result, since as frequency decreases the magnetisation components can follow the field in time and the dynamic transition disappears. This is why the transition is called truely dynamic and discussed in details in earlier studies [1] in the case of Ising model.

\section{SUMMARY}

The classical Heisenberg ferromagnet (uniaxially anisotropic) in presence of a time-varying polarised magnetic field and in contact with a thermal bath is studied by Monte Carlo simulation using Metropolis dynamics. The magnetic field is elliptically polarised and the field vector rotates on XZ - plane. For a very weakly anisotropic (uniaxial) system and particular set of parameter values (amplitudes of field in $\mathrm{x}$ and $\mathrm{z}$ directions) if the system cools down it undergoes successive phase transitions. For a range of values of field amplitude along $\mathrm{x}$-direction, three dynamic phase transitions were observed. Keeping the values of anisotropy strength and amplitude of field along $\mathrm{z}$ direction fixed, the phase transitions were studied for different values of the field amplitude along the $\mathrm{x}$ direction. In a plane formed by the temperature and the ratio of field amplitudes the phase diagram for the multiple dynamic phase transition was drawn. The nature (continuous/discontinuous) of the transitions are investigated by the temperature variations of the Binder cumulant ratio and the distribution of order parameter near the transition points. The finite size study was done to check that the transitions are not artifacts of limited system size. The variances of dynamic order parameter components are studied as a function of temperature taking system size as parameter. This particular study indicates the existence of a diverging length scale at the transition points. The choice of the parameter values, in the present study, are obtained from various trials. In other parameter range the multiple transitions are also possible to observe, however this particular choice gives quite distinct results of the observed multicritical behaviour [18]. Since, the transition across the outermost boundary of the phase diagram is found to be continuous, it will be much interesting to study the scaling behaviour and to estimate the critical exponents. The frequency variations of these multiple dynamic transitions are also studied very briefly. This shows that the qualitative nature of the phase diagram remains unchanged however the transition temperatures are lowered by lowering the frequency of the polarised magnetic field. This is, of course, an expected result experienced from the earlier studies [1], particularly, in the case of the dynamic transition in Ising model.

The dynamic transition in Ising ferromagnet can be explained simply by spin reversal and nucleation [19]. The multiple dynamic transition occurs in Heisenberg ferromagnet possibly due to the coherent spin rotation [13]. To get the clear idea about it one has to study also the 
dynamic configurations of spins in details. The study of the relaxation dynamics of the system in the low anisotropic limit may help to analyse few results.

The alternative methods of studying this multiple dynamic phase transitions in anisotropic Heisenberg ferromagnet driven by polarised magnetic field are: (i) to study Landau-LifshitzGilbert equation of motion [20] with Langevin dynamics, (ii) spin wave analysis of anisotropic Heisenberg ferromagnet in presence of polarised magnetic field.

\section{ACKNOWLEDGMENTS}

The library facility provided by Saha Institute of Nuclear Physics, Calcutta, India, is gratefully acknowledged.

\section{REFERENCES}

1. M. Acharyya, Int. J. Mod. Phys. C 16 (2005) (in press) cond-mat/0508105, and the references therein; See also B. K. Chakrabarti and M. Acharyya, Rev. Mod. Phys. 71, 847 (1999).

2. T. Tome and M. J. de Oliveira, Phys. Rev. A 41, 4251 (1990).

3. M. Acharyya, Phys. Rev. E 56, 1234 (1997); Phys. Rev. E 56, 2407 (1997)

4. S. W. Sides, P. A. Rikvold and M. A. Novotny, Phys. Rev. Lett. 81, 834 (1998)

5. D. C. Mattis, The Theory of Magnetism I: Statics and Dynamics, Springer Series in Solid State Sciences No. 17 (Springer- Verlag, Berlin, 1988).

6. M. Acharyya, Int. J. Mod. Phys. C 14, 49 (2003); Int. J. Mod. Phys. C 12, 709 (2001).

7. T. Yasui et al., Phys. Rev. E 66, 036123 (2002); 67, 019901(E) (2003) See also, Fujiwara et al, Phys. Rev. E 70, 066132 (2004).

8. H. Jang, M. J. Grimson and C. K. Hall, Phys. Rev. E 68, 046115 (2003).

9. Z. Huang, F. Zhang, Z. Chen, Y. Du, Eur. Phys. J. B, 44, 423 (2005)

10. H. Jang, M. J. Grimson and C. K. Hall, Phys. Rev. B 67, 094411 (2003).

11. M. Acharyya, Phys. Rev. E 69, 027105 (2004).

12. D. Stauffer et al., Computer Simulation and Computer Algebra (Springer-Verlag, Heidelberg, 1989);

13. U. Nowak, in Annual Reviews of Computational Physics, edited by D. Stauffer (World Scientific, Singapore, 2001), Vol. 9, p. 105; D. Hinzke and U. Nowak, Phys. Rev. B 58, 265 (1998).

14. N. Metropolis, A. W. Rosenbluth, M. N. Rosenbluth, A. H. Teller, E. Teller, J. Chem. Phys. 21, 1087 (1953). 
15. M. Krech and D. P. Landau, Phys. Rev. B 60, 3375 (1999)

16. K. F. Riley, M. P. Hobson, and S. J. Bence, in Mathematical Methods for Physics and Engineering, Cambridge University Press, (2004) pp. 1179

17. K. Binder and D. W. Heermann, in Monte Carlo Simulation in Statistical Physics, Springer Series in Solid-State Sciences, (Springer, New York, 1997); D. P. Landau and K. Binder, in A guide to Monte Carlo Simulations in Statistical Physics (Cambridge University Press, Cambridge, 2000), pp. 145.

18. P. M. Chaikin and T. C. Lubensky, in Principles of Condensed Matter Physics, Cambridge University Press, (2004) pp. 172-187.

19. M. Acharyya and D. Stauffer, Eur. Phys. J. B 5, 571 (1998)

20. D. Hinzke, U. Nowak and K. D. Usadel, in Structure and Dynamics of Heterogeneous Systems, Eds. P. Entel and D. E. Wolf ( World Scientific, Singapore, 1999 ), pp. 331-337. 
Figure 1: The phase diagram in $T-h_{0 x}$ plane for the multiple dynamic transitions for $D=0.2$ $f=0.02$ and $h_{0 z}=1.0$. C.T. stands for continuous transition and D.T. stands for discontinuous transition. The natures (continuous/discontinuous) of the transitions across the different phase boundary are marked by C.T and D.T.

Figure 2: The temperature variations of different dynamical quantities for $f=0.02, D=0.2$, $h_{0 x}=0.3$ and $h_{0 z}=1.0$. (A) The magnitudes of components of dynamic order parameter $Q_{x}$, $Q_{y}$ and $Q_{z}$. Vertical lines are errorbars. Note that the sizes of the errorbars become maximum near the transition points. (B) derivatives of dynamic order parameters, (C) the dynamic energy and the (D) dynamic specific heat. Solid lines are guide to the eye. The vertical arrows in $(\mathrm{C})$ and $(\mathrm{D})$ indicates the transition points. The maximum error in estimating the transition temperature is 0.01 .

Figure 3: The temperature variations of dynamic specific heat for $f=0.02, D=0.2, h_{0 z}=1.0$. (A) for $h_{0 x}=0.1$ and (B) for $h_{0 x}=0.5$. Solid lines are guide to the eye. The maximum error in estimating the transition temperature is 0.01 .

Figure 4: The temperature variations of (A) dynamic specific heat and (B) magnitudes of the order parameter components. For $f=0.02, D=0.2, h_{0 x}=0.0$ and $h_{0 z}=1.0$. Vertical lines are errorbars. Note that the sizes of the errorbars becomes maximum near the transition points. Solid line in (A) is guide to the eye.

Figure 5: The unnormalised distributions of the magnitudes of dynamic order parameter component $Q_{y}$ for different temperatures. Here, $f=0.02, D=0.2, h_{0 x}=0.7, h_{0 z}=1.0$ and $f=0.02$.

Figure 6: The unnormalised distributions of the magnitudes of the dynamic order parameter component $Q_{y}$ for different temperatures. Here, $D=0.2, h_{0 x}=0.7, h_{0 z}=1.0$ and $f=0.02$.

Figure 7: The temperature variation of the Binder cumulant ratio $U_{y}$ near the transition points. Here, $D=0.2, h_{0 x}=0.7, h_{0 z}=1.0$ and $f=0.02$.

Figure 8: The temperature variation of the Binder cumulant ratio $U_{z}$ near the transition point. Here, $D=0.2, h_{0 x}=0.3, h_{0 z}=1.0$ and $f=0.02$.

Figure 9: Temperature variations of derivatives of dynamic order parameter components for different system sizes $\left(L=10,20\right.$ and 30) for $f=0.02, D=0.2, h_{0 x}=0.3$ and $h_{0 z}=1.0$. (A) $d Q_{x} / d T$ versus $T$. (B) $d Q_{y} / d T$ versus $T$. (C) $d Q_{z} / d T$ versus $T$. Solid lines are guide to the eye.

Figure 10: Temperature variations of dynamic specific heat for different system sizes $(L=10$, 20 and 30) for $f=0.02, D=0.2, h_{0 x}=0.3$ and $h_{0 z}=1.0$. Solid lines are guide to the eye. 
Figure 11: Temperature variations of variances of the dynamic order parameter components for different system sizes $(L=10,20$ and 30$)$ for $D=0.2, h_{0 x}=0.3$ and $h_{0 z}=1.0$. (A) $L^{3} \delta Q_{x}^{2}$ versus $T$. (B) $L^{3} \delta Q_{y}^{2}$ versus $T$. (C) $L^{3} \delta Q_{z}^{2}$ versus $T$. Solid lines are guide to the eye.

Figure 12: Temperature variations of the (A) magnitudes of dynamic order parameter components (B) the derivatives of the order parameter components. Here, $D=0.2, h_{0 x}=0.3$, $h_{0 z}=1.0$ and $f=0.01$.

Figure 13: Temperature variations of the (A) magnitudes of dynamic order parameter components (B) the derivatives of the order parameter components. Here, $D=0.2, h_{0 x}=1.5$, $h_{0 z}=1.0$ and $f=0.01$. 


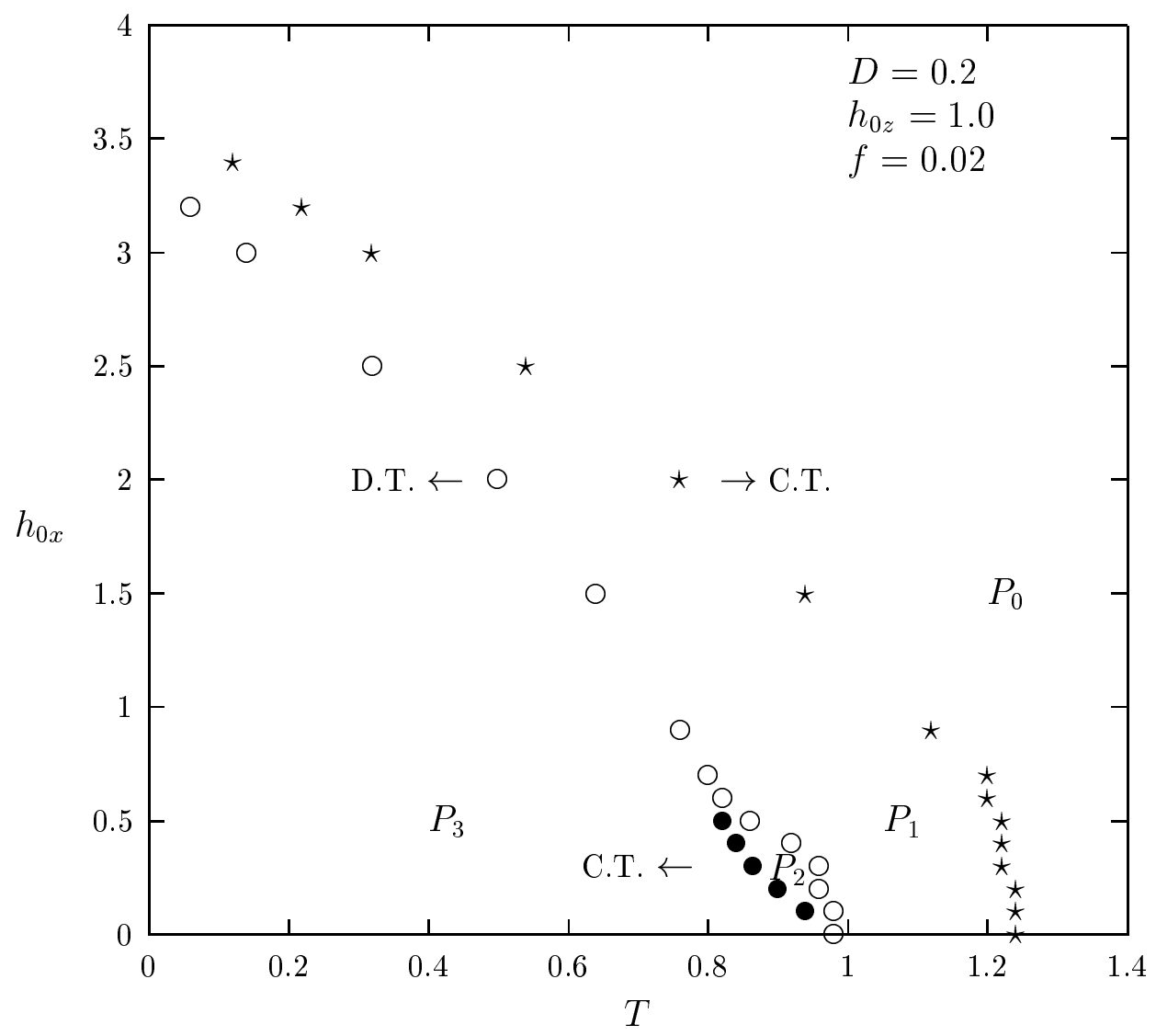

Fig.1 


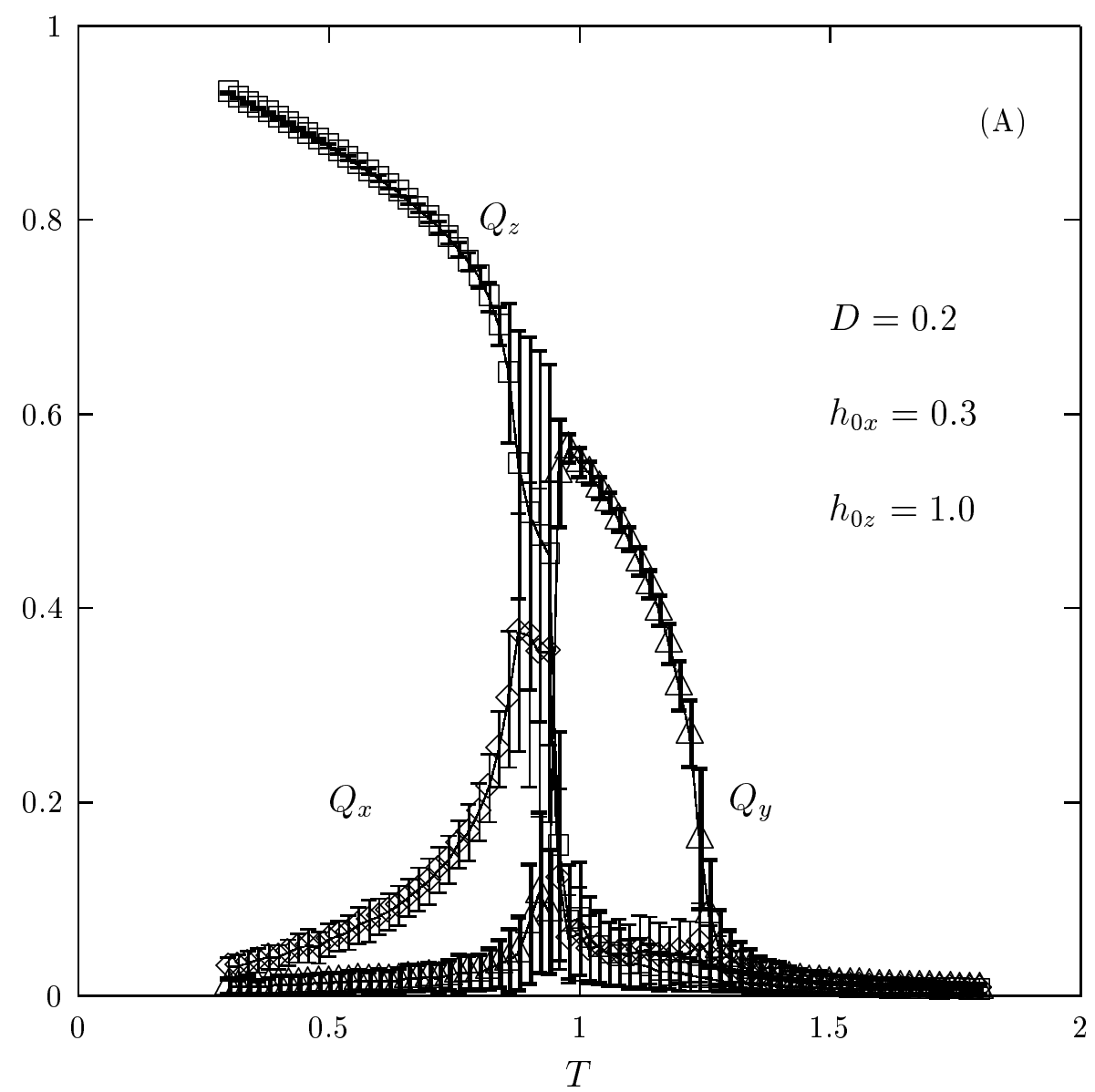

Fig.2a 


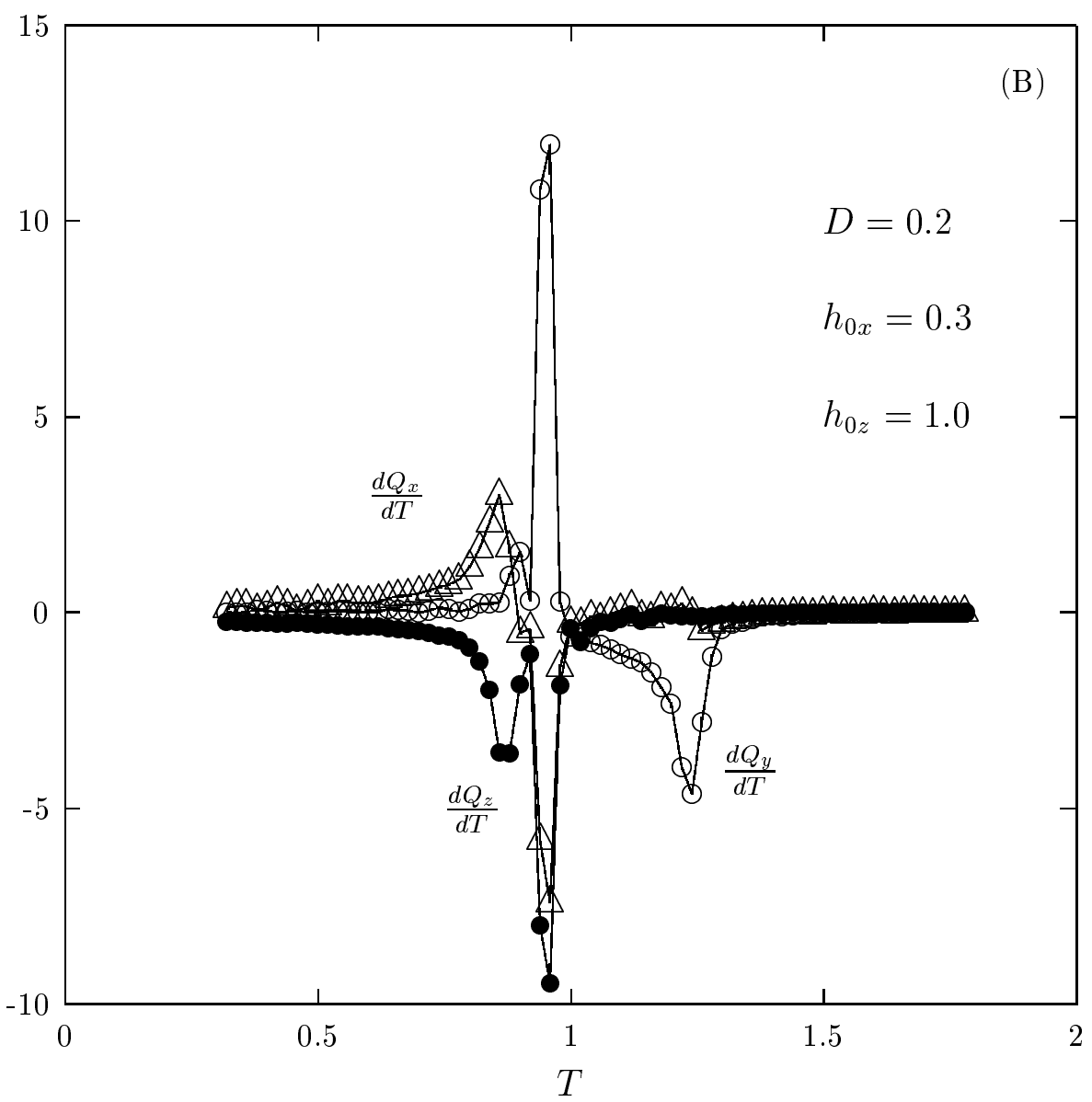

Fig.2b 


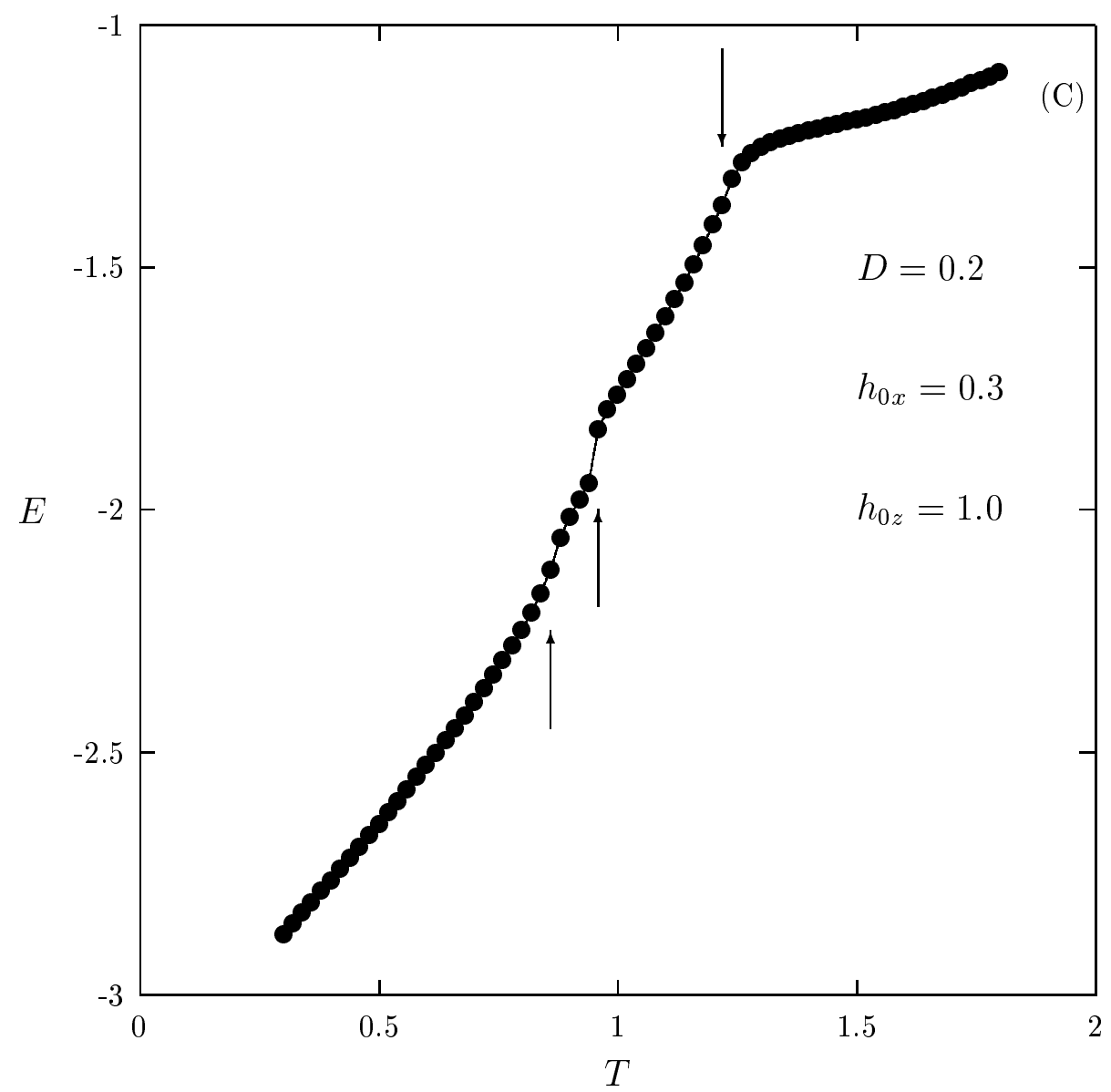

Fig.2c 


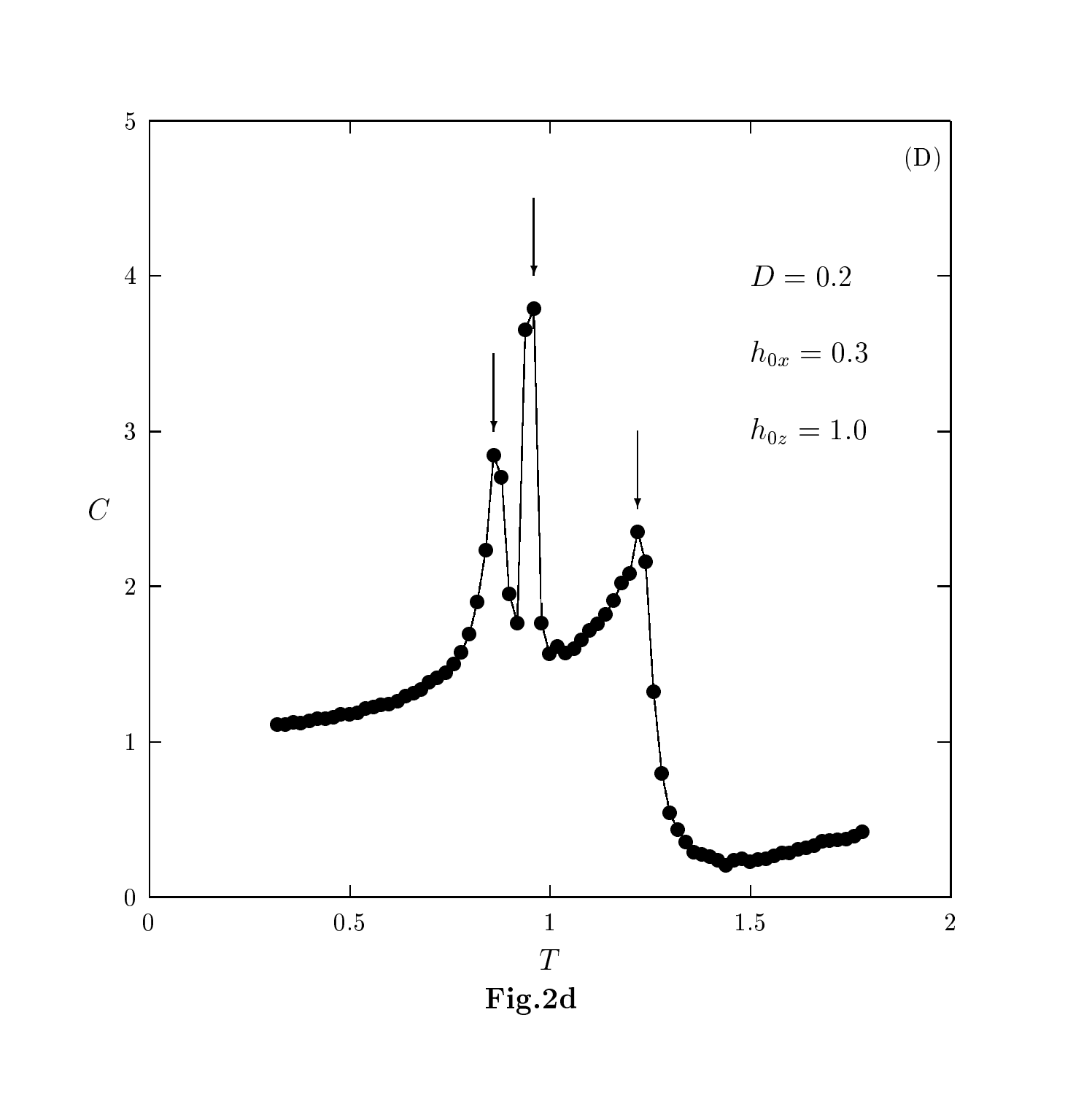



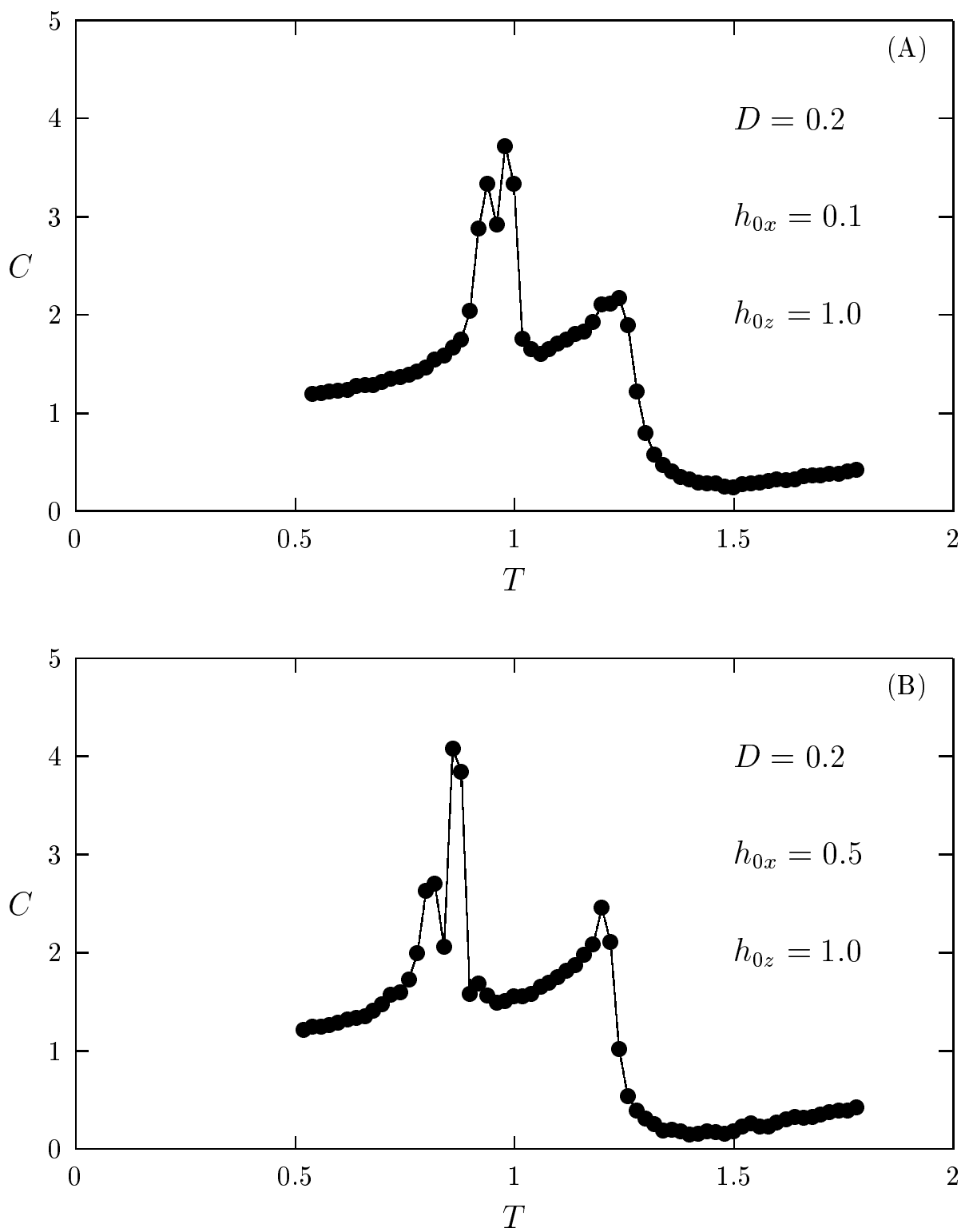

Fig. 3 


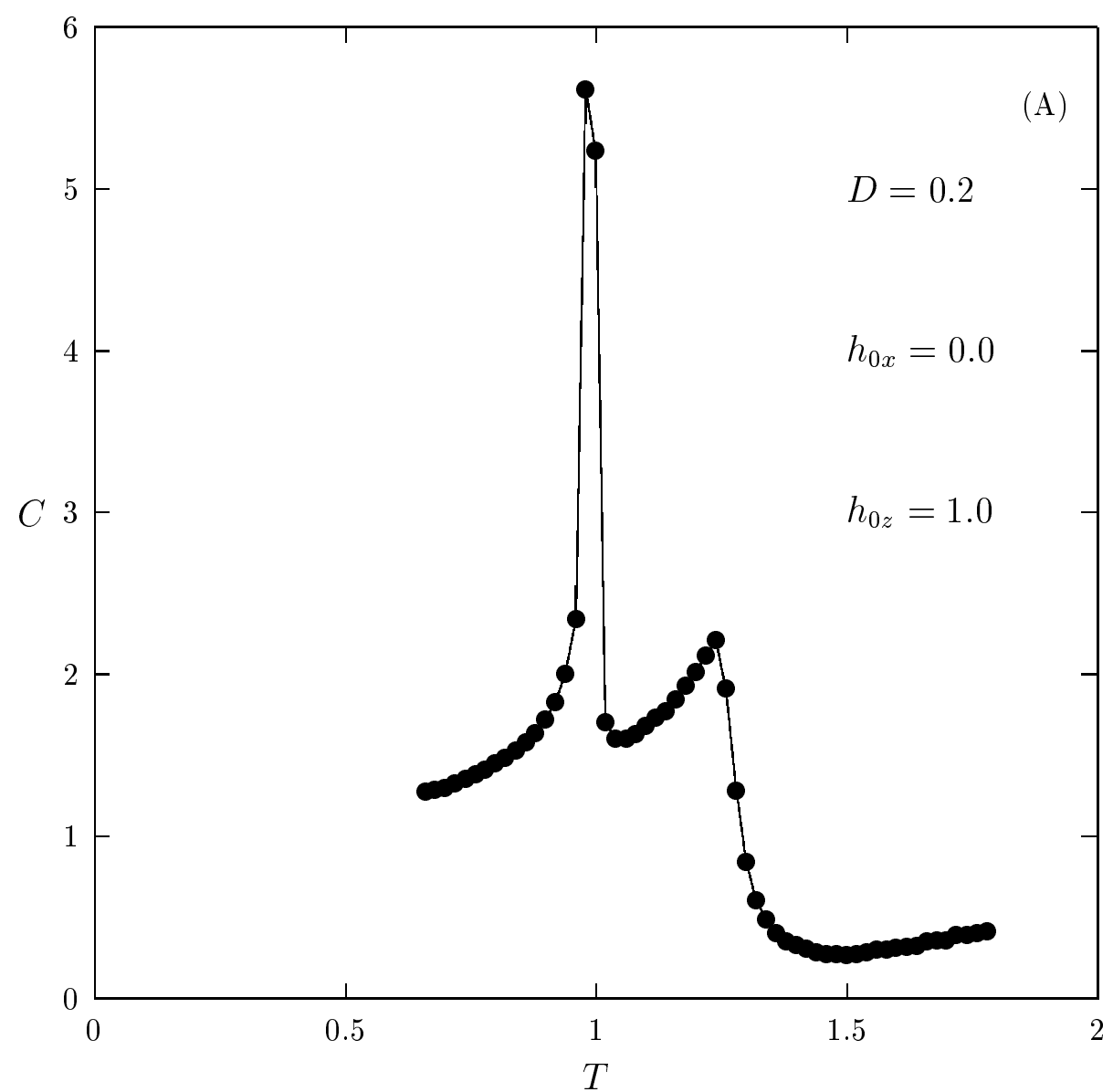

Fig.4a 


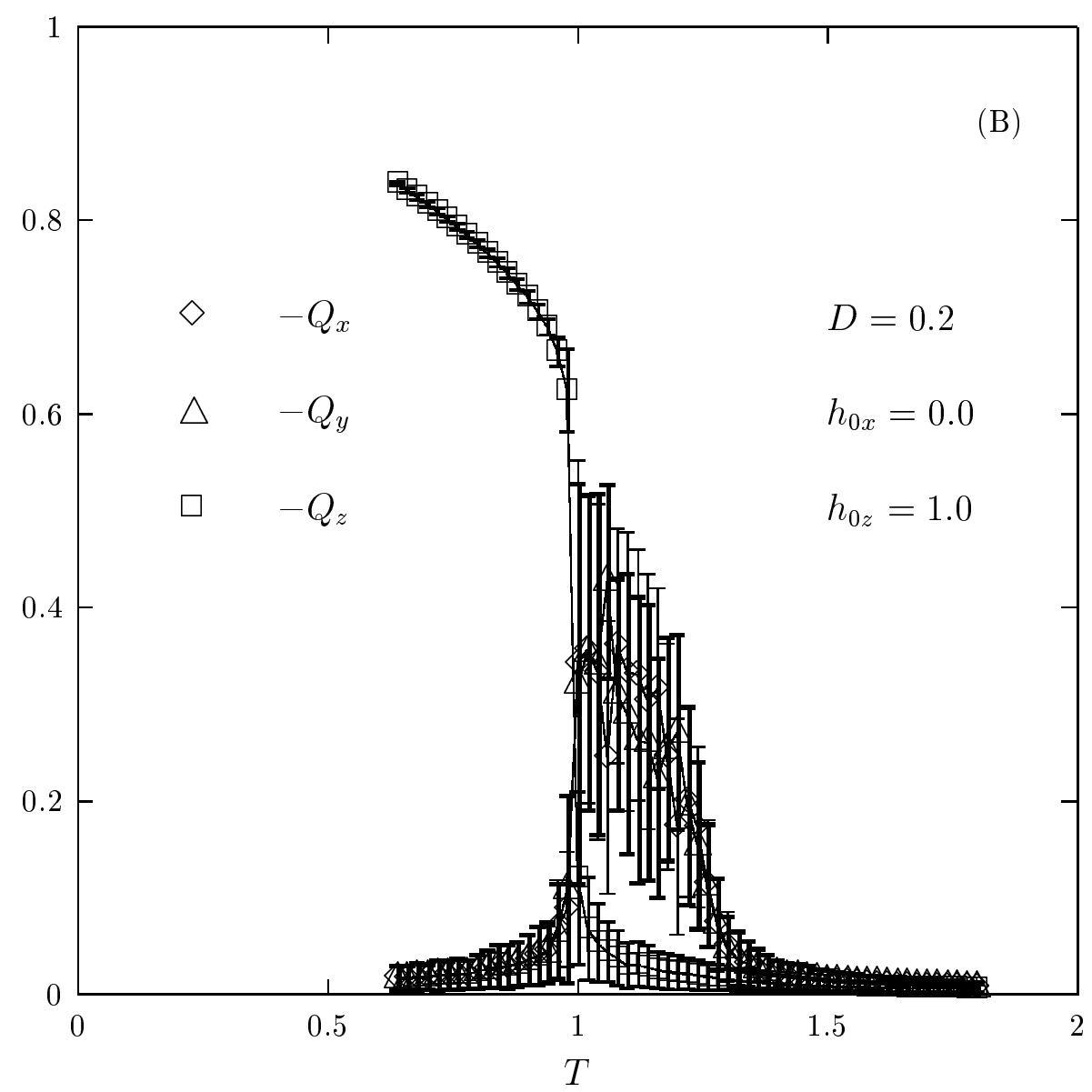

Fig.4b 

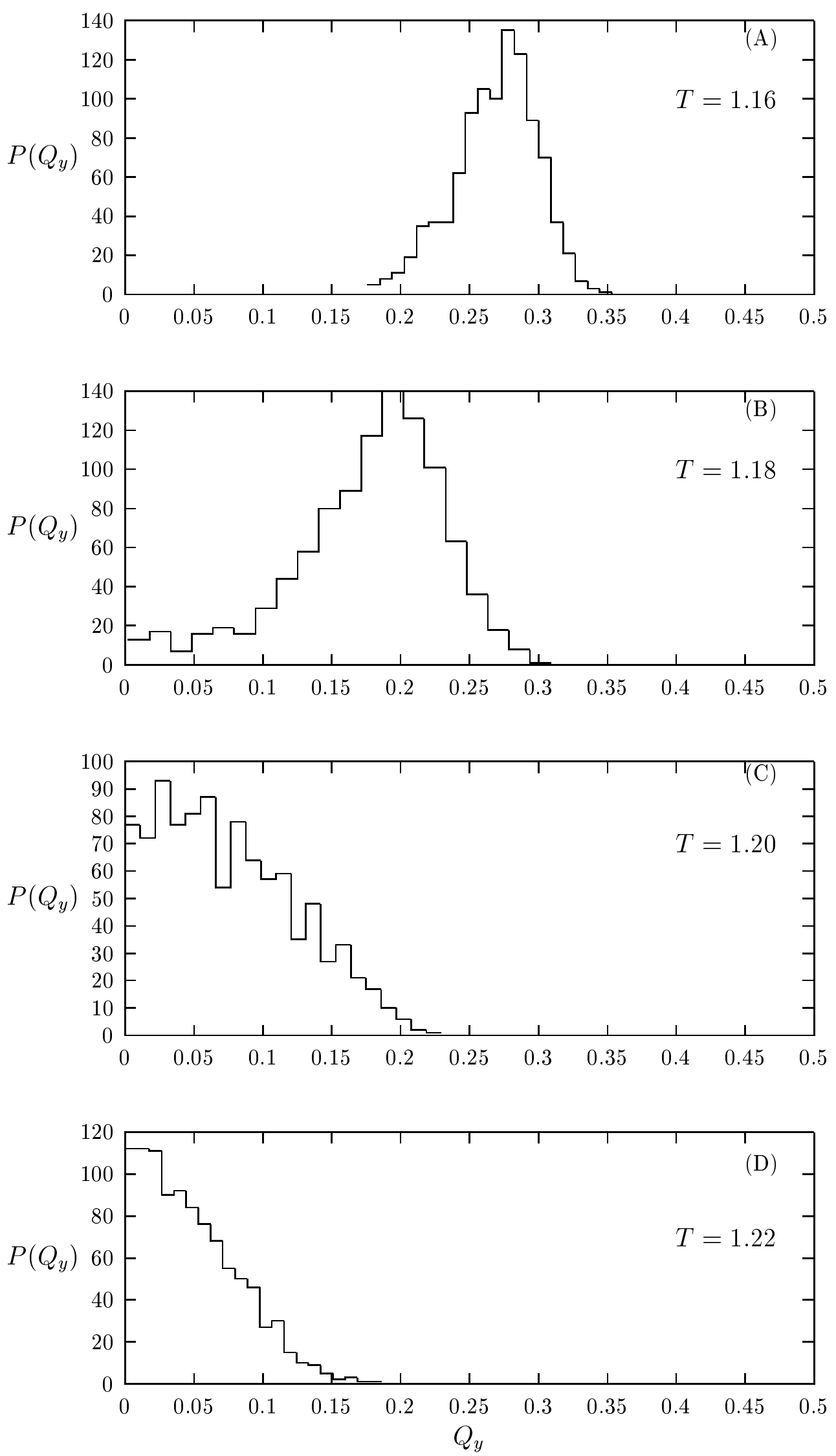

Fig.5 

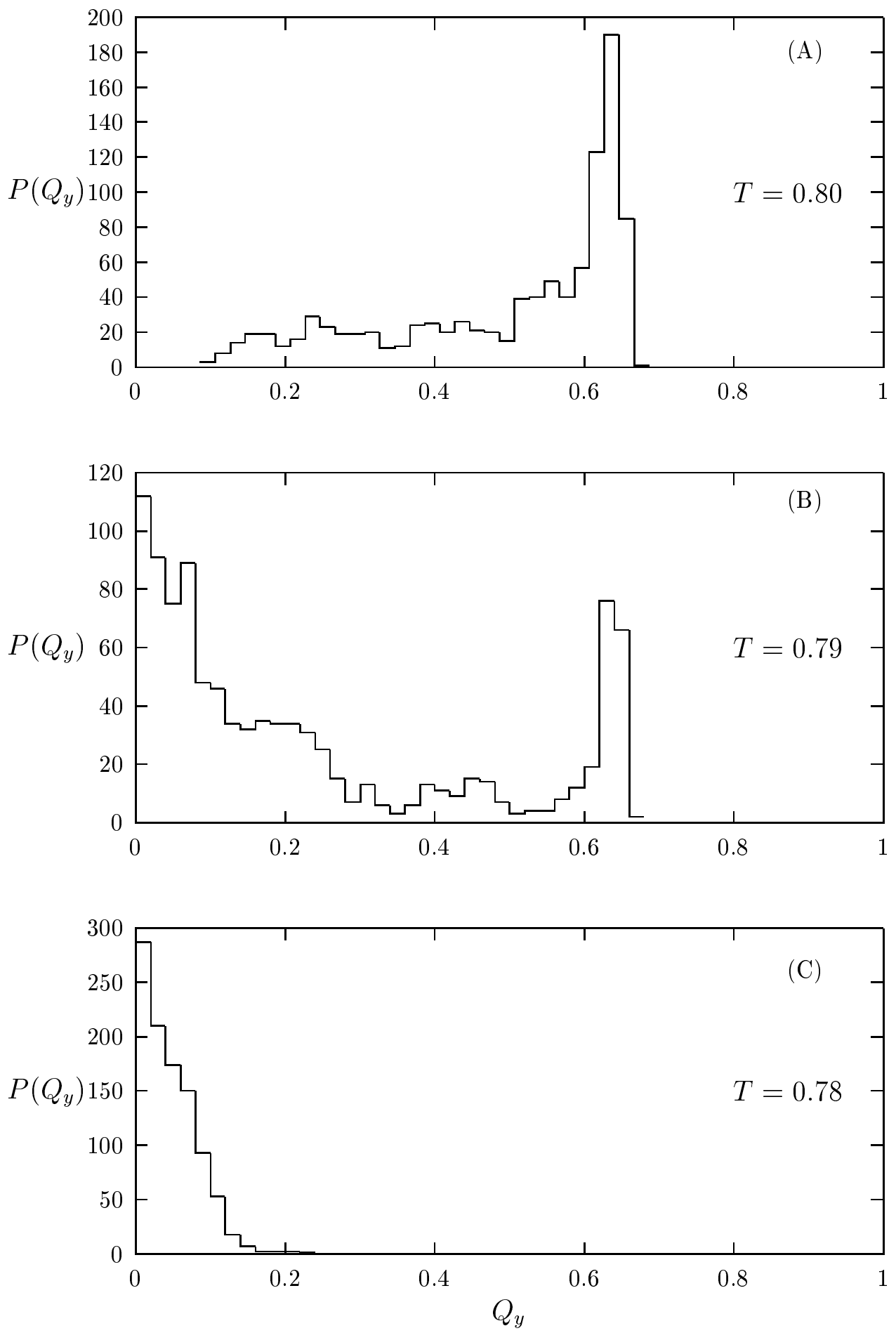

Fig.6 


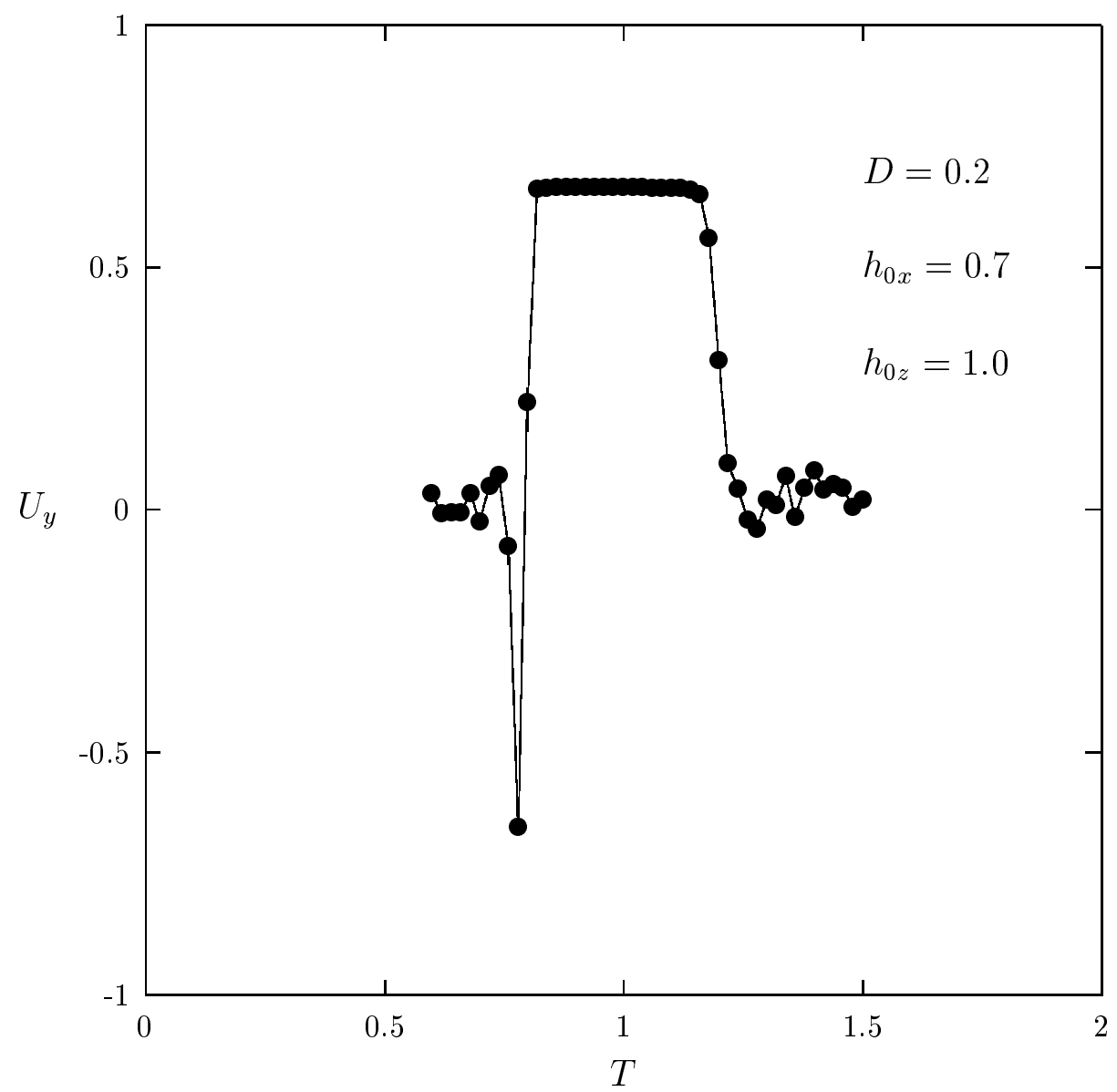

Fig. 7 


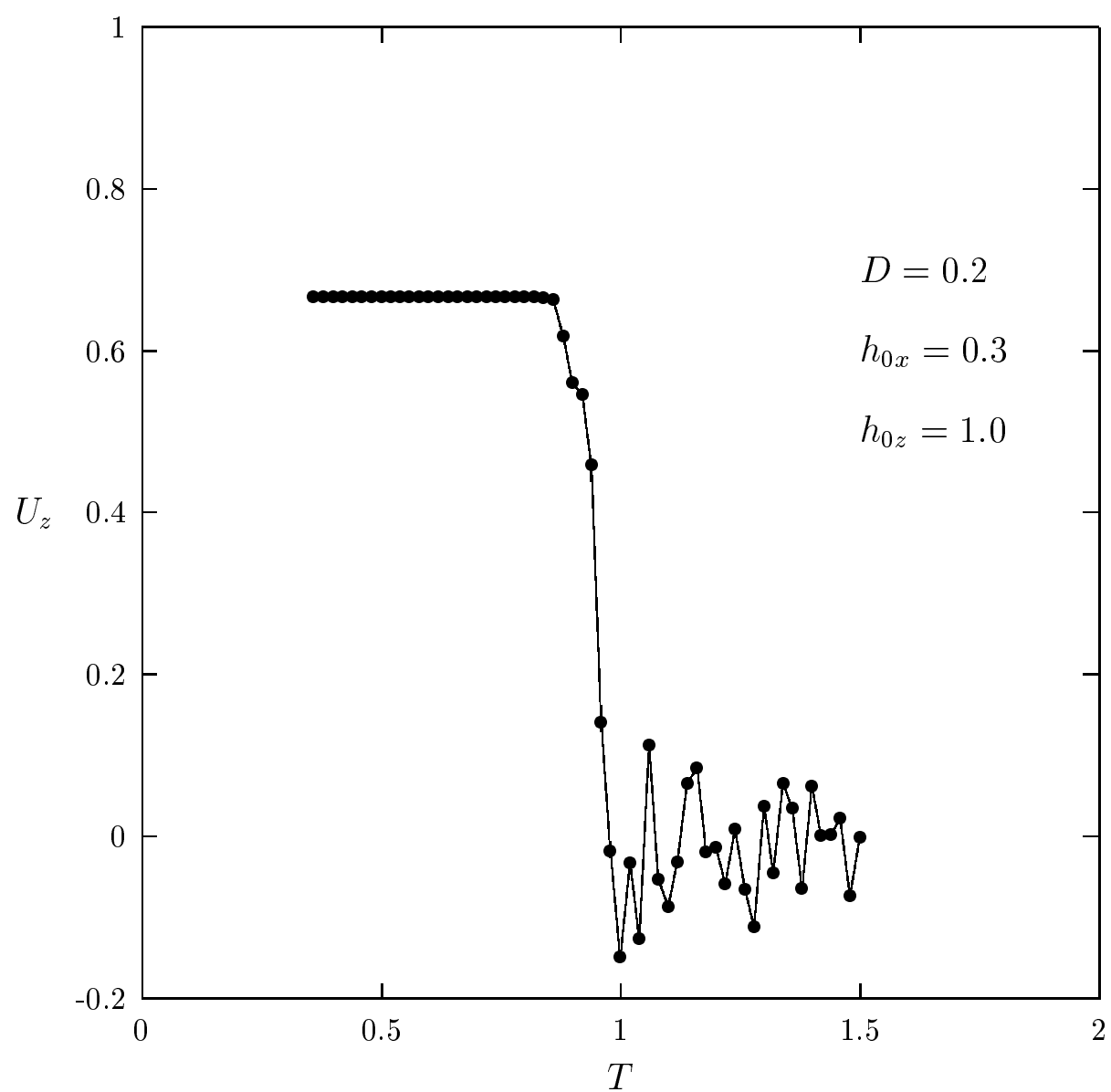

Fig. 8 

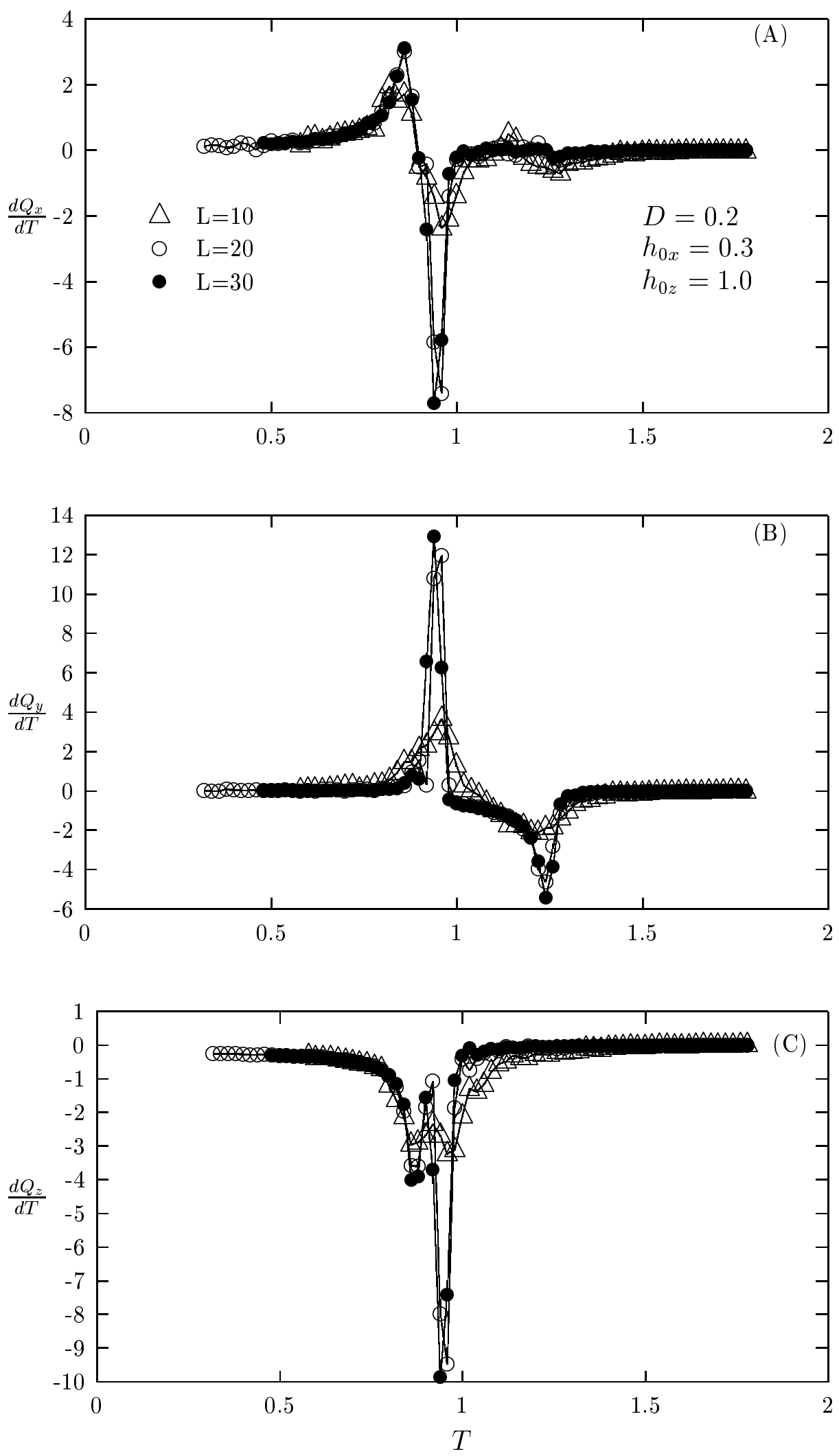

Fig.9 


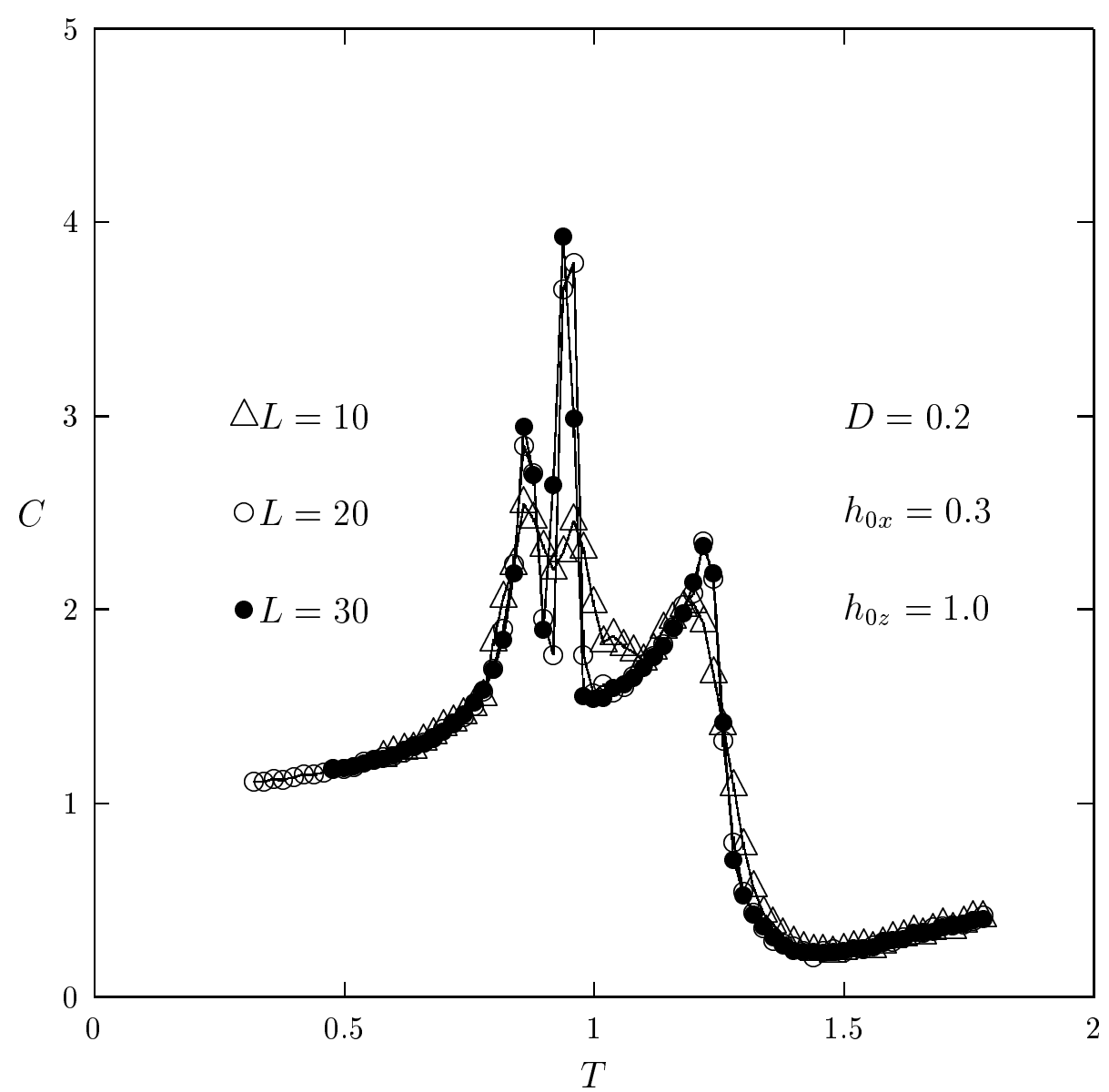

Fig.10 

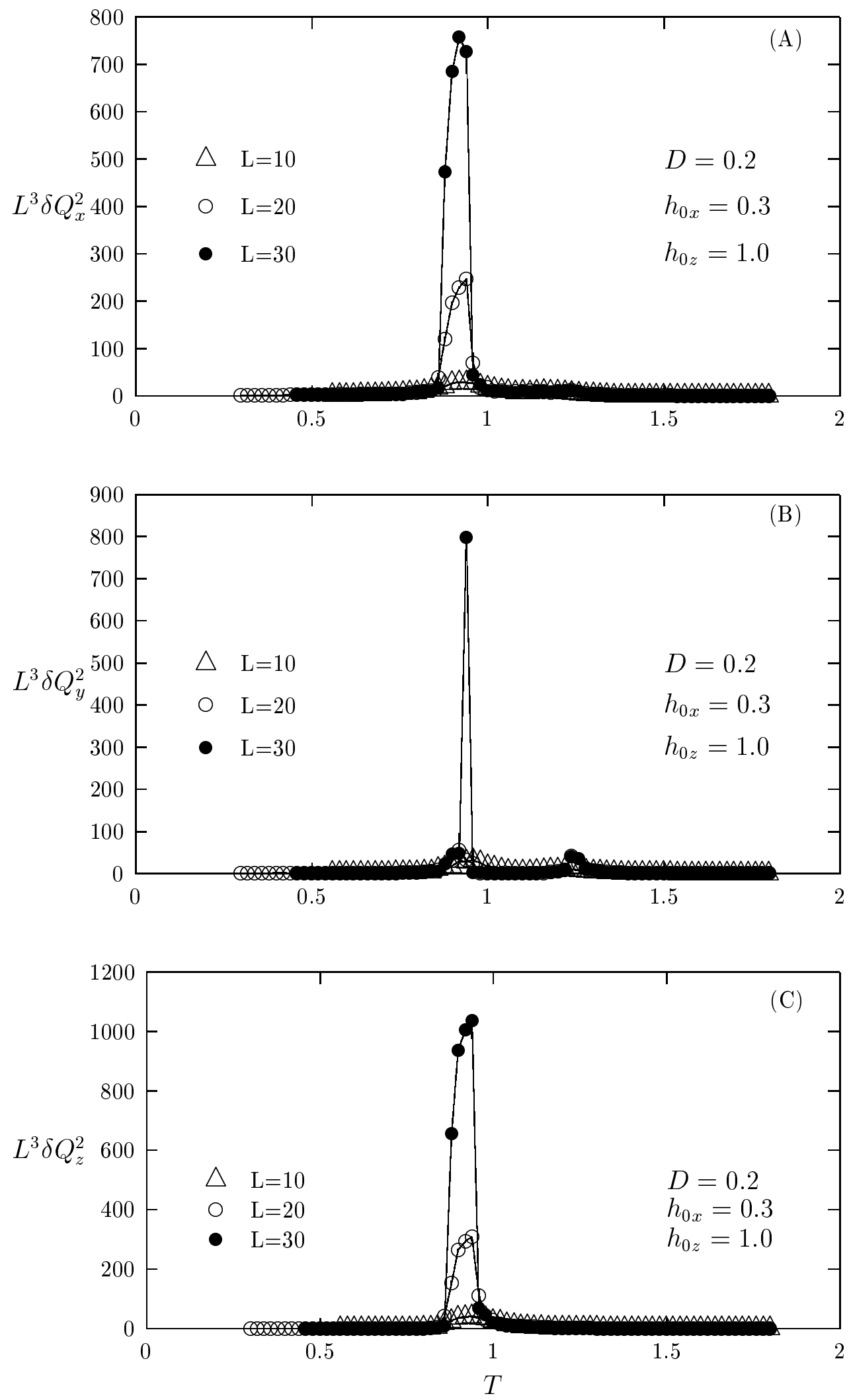

Fig.11 

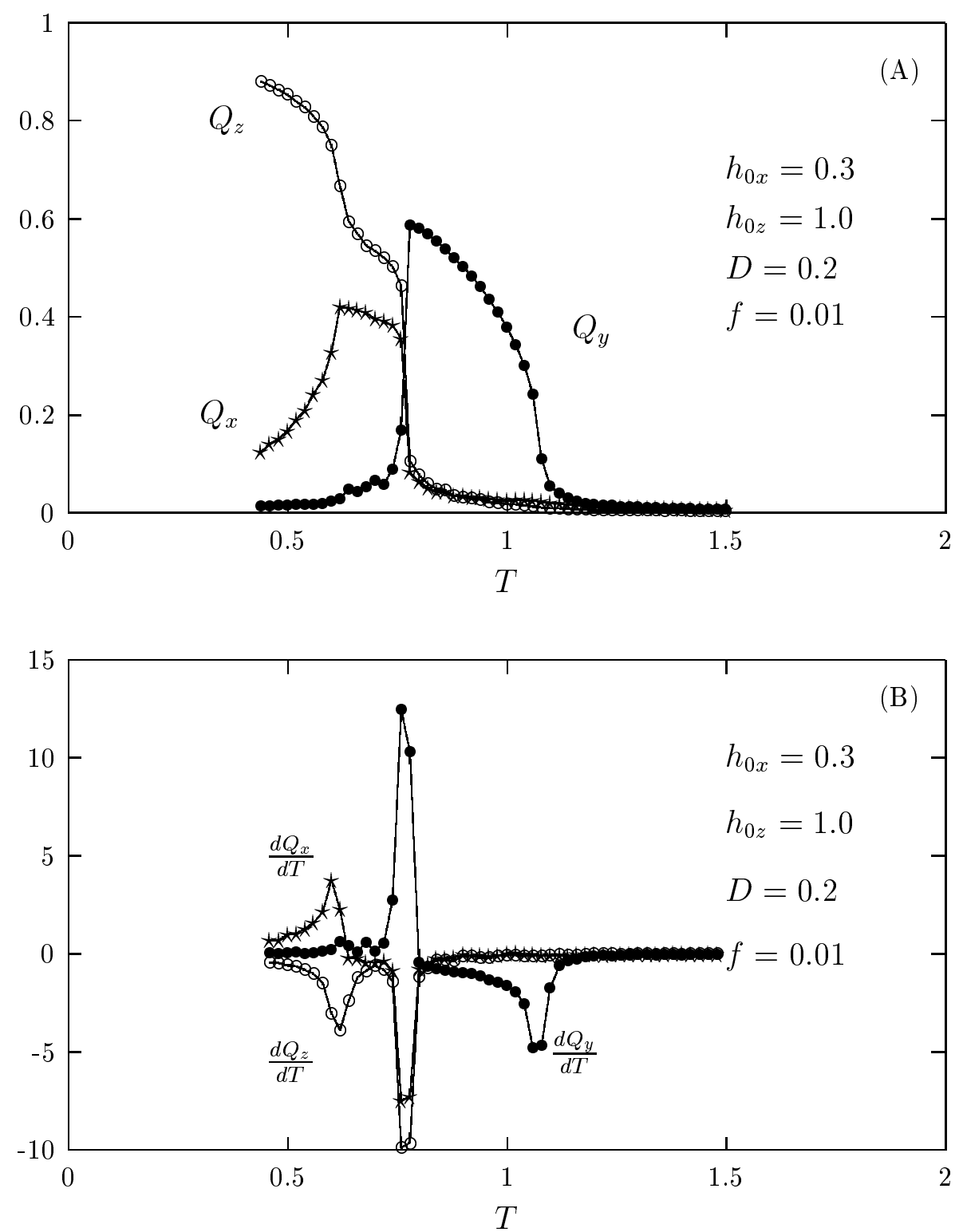

Fig.12 

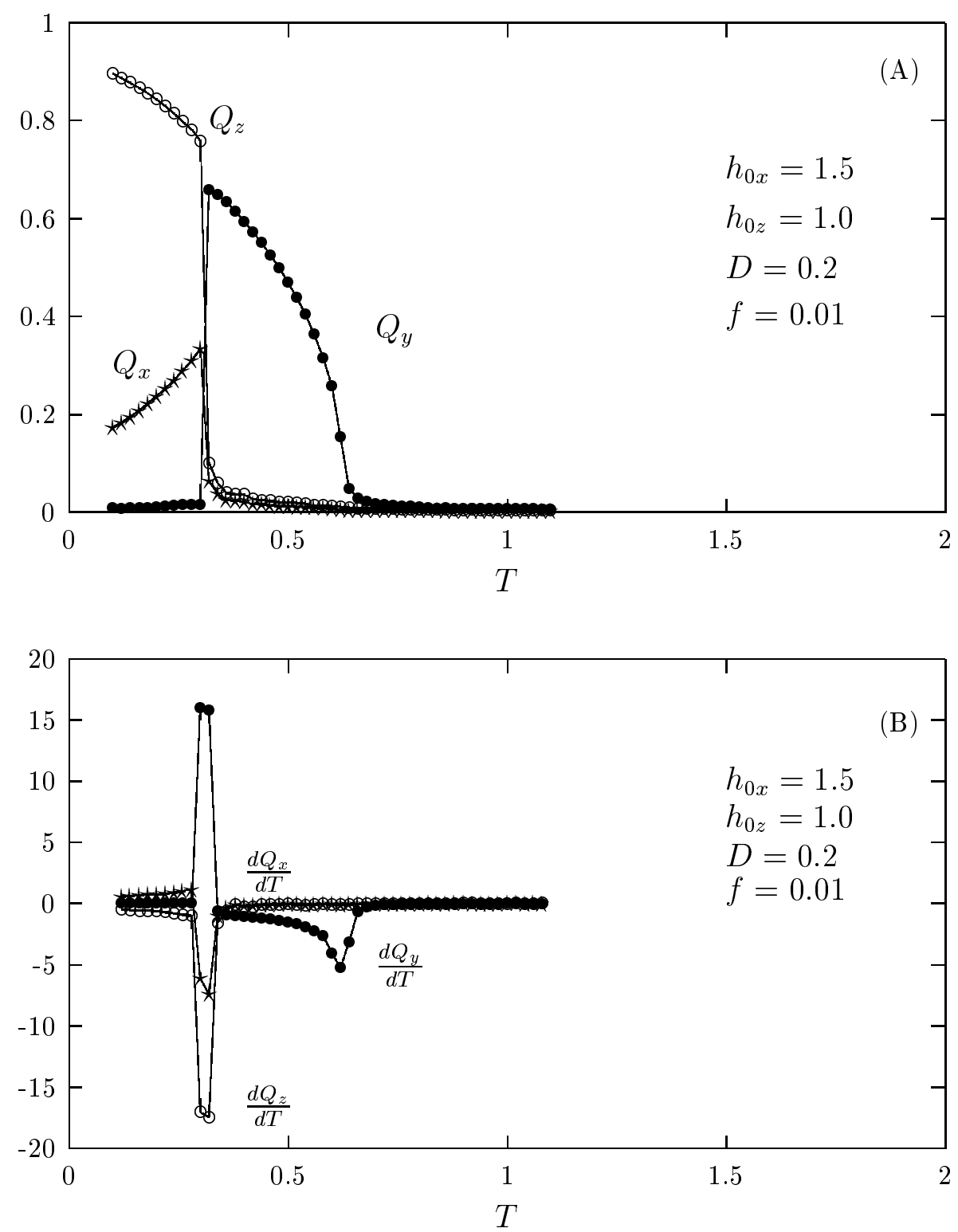

Fig.13 